\title{
Hesitant T-spherical Dombi fuzzy aggregation operators and their applications in multiple criteria group decision-making
}

\author{
Faruk Karaaslan ${ }^{1}$ D $\cdot$ Abdulrasool Hasan Sultan Al-Husseinawi ${ }^{1}$
}

Received: 28 June 2021 / Accepted: 28 January 2022 / Published online: 20 February 2022

(c) The Author(s) 2022

\begin{abstract}
A hesitant fuzzy (HF) set is an extension of the fuzzy sets and a T-spherical fuzzy set (T-SFS) is a generalization of the spherical fuzzy set (SFS). HF set has a significant role for modelling disagreements of the decision-makers over membership degree of an element. Also, T-SFS is quite effective in the modelling of the uncertainty for decision-making (DM) problems. In this paper, we define the concept of hesitant T-spherical fuzzy (HT-SF) set (HT-SFS) by combining concepts of HF set and T-SFS, and present some set-theoretical operations of HT-SFSs. We also develop the Dombi operations on HT-SFSs. We present some aggregation operators based on Dombi operators, including hesitant T-spherical Dombi fuzzy weighted arithmetic averaging operator, hesitant T-spherical Dombi fuzzy weighted geometric averaging operator, hesitant T-spherical Dombi fuzzy ordered weighted arithmetic averaging operator, and hesitant T-spherical Dombi fuzzy ordered weighted geometric averaging operator, and investigate some properties of them. In addition, we give a multi-criteria group decision-making method and algorithm of the proposed method under the hesitant T-spherical fuzzy environment. To show the process of proposed method, we present an example related to the selection of the most suitable person for the assistant professorship position in a university. Besides this, we present a comparative analysis with existing operators to reveal the advantages and authenticity of our technique.
\end{abstract}

Keywords Hesitant fuzzy set · Spherical fuzzy set $\cdot$ T-spherical fuzzy set $\cdot$ Dombi operators $\cdot$ Decision-making

\section{Introduction}

The notion of fuzzy set (FS) was defined by Zadeh [1] in 1965 to model some problems involving uncertainty. The FS has found application in many different fields such as computer science, medical science, clustering, robotic, optimization, and data mining. For example, Gadekallu and Gao [2] proposed a model using an approach based on rough sets for reducing the attributes and fuzzy logic system for classification for prediction of the Heart and Diabetes diseases; Sankaran et al. [3] proposed a method to provide a multi-path and multi-constraint Qualify of Service based on Reliable Fuzzy and Heuristic Concurrent Ant Colony Optimization. These studies are two example made in 2021 related to application of fuzzy logic and sets.

Faruk Karaaslan

karaaslan.faruk@gmail.com

Abdulrasool Hasan Sultan Al-Husseinawi rassolhussanawi55@yahoo.com

1 Department of Mathematics, Faculty of Sciences, Cankiri Karatekin University, 18100 Cankiri, Turkey
Zadeh [1] characterize an FS by the membership function of which codomain is interval $[0,1]$. In an FS, if the membership degree (MD) of an element is $\mu$, then its nonmembership degree (NMD) is $1-\mu$. Namely, in an FS, hesitation degree of an element is accepted as " 0 ". However, this perspective has some constraints. To overcome with this constraints, Atanassov [4] introduced the concept of intuitionistic FS (IFS) as a generalization of FSs. An IFS is defined by assigning two values from the range $[0,1]$, named MD $\mu$ and NMD $\nu$, under the condition $\mu+v \leq 1$ for all elements of the working universe. However, this set is not useful when $\mu+v>1$. Therefore, Yager [5,6] defined the Pythagorean FS (PyFS) as an extension of IFS under condition $\mu^{2}+v^{2} \leq 1$. Another extension of IFS is Picture FS (PFS) defined by Cuong [7,8]. PFS is a useful tool for representing human opinion, because a PFS can model judgments about an object or idea using degrees of yes, abstention, no, and rejection. A PFS is identified three degrees of an element, called MD $(\mu)$, abstinence degree (AD) or neutral degree $(\gamma)$, and NMD ( $v)$ with the condition $0 \leq \mu+\gamma+v \leq 1$. Although PFS has wide applications in some field such as decision-making (DM) [9-15], similarity measure [16-20], 
correlation coefficient [21,22], and clustering [23,24], it is not sufficient in modelling some problems when $\mu+\gamma+v>1$. For this reason, Gungogdu and Kahraman [25,26] inaugurated the design of spherical FS (SFS) which is an extension of PFS satisfying the condition $0 \leq \mu^{2}+\gamma^{2}+v^{2} \leq 1$. They also studied on SFS operations and applications of this set in DM problems. Kahraman et al. [25] proposed a DM method by integrating the SFS and TOPSIS method, and gave an application of the proposed method in the selection of hospital location. Mahmood et al. [27] defined the T-spherical FS (T-SFS) as an extension of the SFS with condition $0 \leq$ $\mu^{q}+\gamma^{q}+v^{q} \leq 1$ and gave some applications in medical diagnosis and decision-making problems of T-SFS and SFS. Ullah et al. [28] introduced the similarity measures for T-SFSs and presented an application in pattern recognition. Garg et al. [29] presented improved interactive aggregation operators for T-SFSs and studied on operational laws of these operators. Ullah et al. [30] described some ordered weighted geometric (OWG) and hybrid geometric (HG) operators, and gave a numerical example involving multi-attribute decisionmaking (MADM) problem. Ullah et al. [31] introduced the concept of interval-valued T-SFS (IVT-SFS) and basic operations of them. They also defined two aggregation operators including weighted averaging and weighted geometric operators for IVT-SFS, and presented an MCDM method. Liu et al. [32] pointed out some limitations in operational laws of SFS and T-SFS, and suggested some novel operational laws for SFS and T-SFS. They also introduced Power Muirhead Mean Operator for T-SFS by combining power average operator with Muirhead Mean operator and presented an MAGDM method based on proposed operators. Recently, T-SFS has gained attention of researchers working on MCDM methods, MCGDM methods, and aggregation operators. For example, divergence measure of T-SFSs [33], immediate probabilistic Interactive averaging aggregation operators of T-SFSs [34], T-SF soft sets and their aggregation operators [35], generalized T-SF weighted aggregation operators on neutrosophic sets [36], T-SF Einstein Hybrid Aggregation operators [37], correlation coefficients for T-SFSs [38], T-SF Hamacher aggregation operators [39], complex T-SF aggregation operators [40], and T-spherical Type-2 fuzzy sets [41] are some of them.

The hesitant FS (HFS) is another extension of the FS for modelling the problems in which decision-makers have different opinions about an alternative or element in considered universe. The HFS was defined by Torra and Narukawa in $[42,43]$. To explain the basic idea behind of the concept of the hesitant fuzzy set, we give an example: two decisionmakers discuss the membership grade of an element to a set, and while one of them assigns membership grade 0.7 for the element, the other may assign 0.3. In such cases, making a common decision is difficult. In a such case, the HFS is a useful tool. Because of advantages of HFS, many researchers have been developed multiple decision-making methods and they have presented their applications under HF environment [44-49]. Xia et al. [50] described some HF aggregation operators and developed a group decisionmaking method. Chen et al. [51] interpreted the idea of interval-valued hesitant fuzzy sets (IvHFSs) which is a generalization of HFS. Peng et al. [52] investigated the continuous HF aggregation operators with the aid of continuous OWA operator, and they defined the C-HFOWA operator and CHFOWG operator with their essential properties. They also extended these operators interval-valued HFS. Mu et al. [53] introduced a novel aggregation principle for HF elements (HFE). Amin et al [54] defined some aggregation operators for triangular cubic linguistic hesitant fuzzy sets. Fahmi et al. [55] defined some new operation laws for trapezoidal cubic hesitant fuzzy ( $\mathrm{TrCHF}$ ) numbers and introduced some new aggregation operators. Jiang et al. [56] defined the concept of interval-valued dual HFS, and described aggregation operators under interval-valued dual HF environment based on Hamacher t-norm and t-conorm. Liu et al. [57] introduced the Dombi aggregation operators of interval-valued hesitant fuzzy set based on Dombi t-norm and t-conorm. Some studies related to aggregation operator of HFS, extension of HFS, and decision-making can be found [58-72].

It has been mentioned above that HFSs are an important set structure in modelling problems involving multiple decisionmakers, in terms of revealing the ideas of decision-makers. In an HFS, the HFEs are subsets of the interval [0,1]. In other words, the elements of an HFE express their degree of membership. It is insufficient to express non-membership and neutral status. As mentioned above, TSFS is defined as a generalization of FS, IFS, PyFS, PFS, qROFS, and SFS, and finds application in decision-making problems. Some generalizations of HFSs are available in the literature. To use the advantages of HFSs and T-SFSs together, in this article, we define a novel concept called the hesitant T-spherical fuzzy set (HT-SFS) by combining concepts of HFs and TSFS. In HT-SFS, more than one T-spherical fuzzy value can be assigned to elements of the set containing the elements to be evaluated. T-SFS theory deals only one T-spherical fuzzy value for an element. Therefore, it does not suffice to model problems including disagreements of the opinion of decisionmakers about an element or object. On the other hand, an HT-SFS can handle such situation. We also introduce some aggregation operators based on Dombi operators, including hesitant T-spherical Dombi fuzzy weighted arithmetic averaging (HTSDFWAA) operator, hesitant T-spherical Dombi fuzzy weighted geometric averaging (HTSDFWGA) operator, hesitant T-spherical Dombi fuzzy ordered weighted arithmetic averaging (HTSDFOWAA) operator, and hesitant T-spherical Dombi fuzzy ordered weighted geometric averaging (HTSDFOWGA) operator, and obtain some properties of them. Furthermore, we give a multi-criteria group 
decision-making (MCGDM) method and algorithm of the proposed method under the HT-SF environment. To show the process of of proposed method, we present an example related to the selection of most suitable person for the assistant professorship position in a university. Besides this, we have presented a comparison of the proposed methods with each other and a comparison table of the proposed clusters with other extensions of the FS.

\section{Preliminaries}

This section provides some basic definitions and operations that will be needed in the next sections. First, for the reader's convenience, a table of notations by chapter is given in Table 1.

Definition 1 [25] An SFS $\mathbb{S}$ on a universe $\mathfrak{X}$ is represented as follows:

$\mathbb{S}=\{(x, s(x), i(x), d(x)): x \in \mathfrak{X}\}$,

where $s(x), i(x), d(x) \in[0,1], 0 \leq s^{2}(x)+i^{2}(x)+$ $d(x)^{2} \leq 1$ for all $x \in \mathfrak{X}$. We consider the triplet $(s, i, d)$ as SF number (SFN). Here, $s, i$, and $d$ are the membership degree (MD), abstinence degree (AD), and non-membership degree (NMD) of $x \in \mathbb{S}$, respectively. Further $\pi_{\mathbb{S}}(x)=$ $\sqrt{1-\left(s^{2}(x)+i^{2}(x)+d^{2}(x)\right)}$ is the hesitancy degree of $x$ in $\mathbb{S}$.

Definition 2 [27] A T-SF Set (T-SFS) on $\mathfrak{X}$ is defined as

$T=\{(x, s(x), i(x), d(x)): x \in \mathfrak{X}\}$,

where $s, i, d: \mathfrak{X} \longrightarrow[0,1]$ are membership, abstinence, and non-membership functions with a condition that

$0 \leq s^{n}(x)+i^{n}(x)+d^{n}(x) \leq 1$.

The refusal degree of T-SFS is defined as

$r(x)=\sqrt[n]{1-\left(s^{n}(x)+i^{n}(x)+d^{n}(x)\right)}$,

and the triplet $(s, i, d)$ is called the T-SF number (T-SFN).

Definition $3[42,43]$ Let $\mathfrak{X}$ be a fixed set, a hesitant fuzzy set (HFS) on $\mathfrak{X}$ is in terms of a function that applied to $\mathfrak{X}$ returns of $[0,1]$.The mathematical symbol of HFS

$A=\left\{<x, h_{A}(x)>: x \in \mathfrak{X}\right\}$,

where $h_{A}(x)$ is a set of some values in [0,1], denoting the possible membership degrees of the element $x \in \mathfrak{X}$ to the set A. $h=h_{A}(x)$ is called an HF element (HFE).
From now on, set of all T-spherical fuzzy numbers is denoted by $\Upsilon$.

\section{Hesitant T-spherical fuzzy sets}

In this part, we define the concept of hesitant T-Spherical fuzzy sets and their set-theoretical operations.

Definition 4 Let $\mathfrak{X}$ be a nonempty set. A hesitant T-SFS (HTSFS) over $\mathfrak{X}$ denoted by $\mathbb{T}_{H}$ is defined as follows:

$\mathbb{T}_{H}=\{(x, \mathfrak{h}(x)): \mathfrak{h}(x) \subseteq \Upsilon, x \in \mathfrak{X}\}$.

Here, $\mathfrak{h}(x)=\mathfrak{h}$ is collection of T-SFNs and $\mathfrak{h}$ is called HT-SF element (HT-SFE). The number of elements of an HT-SFE is called length of HT-SFE $\mathfrak{h}$ and denoted by $\ell_{\mathfrak{h}}$.

In other words, an HT-SFS is collection of HT-SFEs.

Example 1 Let us consider a set $\mathfrak{X}=\left\{x_{1}, x_{2}, x_{3}, x_{4}\right\}$. Then, for $t=3$, we can write an HT-SFS $\mathbb{T}$ as follows:

$$
\begin{aligned}
\mathbb{T} & =\left\{\left(x_{1},\{(0.6,0.8,0.4),(0.4,0.5,0.9),(0.6,0.2,0.7)\}\right),\right. \\
& \left(x_{2},\{(0.3,0.9,0.5),(0.2,0.4,0.7)\}\right) \\
& \left(x_{3},\{(0.9,0.3,0.2),(0.6,0.6,0.6),(0.4,0.3,0.8)\right. \\
& \left.(0.5,0.7,0.6)\}),\left(x_{4},\{(0.7,0.2,0.2)\}\right)\right\}
\end{aligned}
$$

Definition 5 Let $\mathfrak{h}$ be an HT-SFE. Then, score value of HT$\mathrm{SFE} \mathfrak{h}$ denoted by $\mathrm{SV}(\mathfrak{h})$ is defined as

$S V(\mathfrak{h})=\frac{1}{\ell_{\mathfrak{h}}} \sum_{k=1}^{\ell_{\mathfrak{h}}}\left(s_{k}^{n}-d_{k}^{n}\right)$

for some positive integer $n$. Here, $\operatorname{SV}(\mathfrak{h}) \in[-1,1]$.

Definition 6 Let $\mathfrak{h}$ be an HT-SFE. Then, accuracy value of HT-SFE $\mathfrak{h}$ denoted by $\mathrm{AV}(\mathfrak{h})$ is defined as

$A V(\mathfrak{h})=\frac{1}{\ell_{\mathfrak{h}}} \sum_{k=1}^{\ell_{\mathfrak{h}}}\left(s_{k}^{n}+i_{k}^{n}+d_{k}^{n}\right)$

for the positive integer $n$. Here, $\operatorname{AV}(\mathfrak{h}) \in[0,1]$.

Definition 7 Let $\mathfrak{h}_{1}$ and $\mathfrak{h}_{2}$ be two HT-SFEs, SV(h $\left(\mathfrak{h}_{1}\right)$ and $\mathrm{SV}\left(\mathfrak{h}_{2}\right)$ are the score values of $\mathfrak{h}_{1}$ and $\mathfrak{h}_{2}$, respectively, and $\operatorname{AV}\left(\mathfrak{h}_{1}\right)$ and $\operatorname{AV}\left(\mathfrak{h}_{2}\right)$ are the accuracy values of $\mathfrak{h}_{1}$ and $\mathfrak{h}_{2}$, respectively. Then

1. If $\operatorname{SV}\left(\mathfrak{h}_{1}\right)<\operatorname{SV}\left(\mathfrak{h}_{2}\right)$ then $\mathfrak{h}_{1}<\mathfrak{h}_{2}$

2. If $\operatorname{SV}\left(\mathfrak{h}_{1}\right)>\operatorname{SV}\left(\mathfrak{h}_{2}\right)$ then $\mathfrak{h}_{1}>\mathfrak{h}_{2}$

3. If $\operatorname{SV}\left(\mathfrak{h}_{1}\right)=\operatorname{SV}\left(\mathfrak{h}_{2}\right)$, there are three cases 
Table 1 Frequently used notations in "Preliminaries", "Hesitant T-spherical fuzzy sets", and "Hesitant T-spherical Dombi fuzzy aggregation operators"

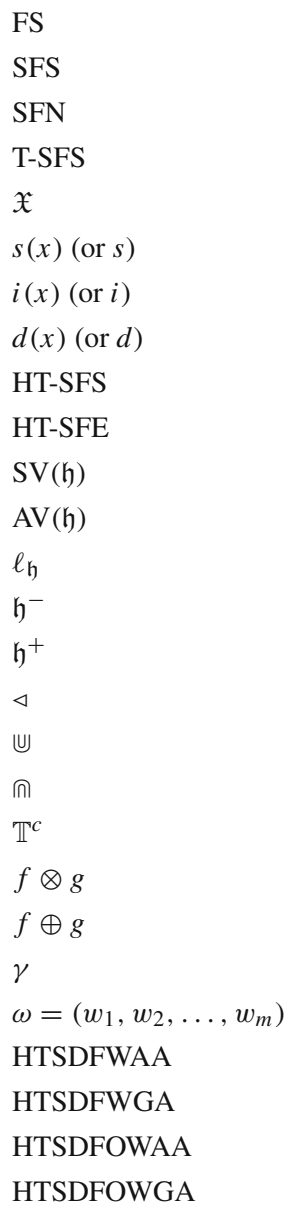

(a) If $\operatorname{AV}\left(\mathfrak{h}_{1}\right)<\operatorname{AV}\left(\mathfrak{h}_{2}\right)$, then $\mathfrak{h}_{1}<\mathfrak{h}_{2}$

(b) If $\operatorname{AV}\left(\mathfrak{h}_{1}\right)>\operatorname{AV}\left(\mathfrak{h}_{2}\right)$, then $\mathfrak{h}_{1}>\mathfrak{h}_{2}$

(c) If $A V\left(\mathfrak{h}_{1}\right)=A V\left(\mathfrak{h}_{2}\right)$, then $\mathfrak{h}_{1}=\mathfrak{h}_{2}$.

Example 2 Let us consider HT-SFEs $\mathfrak{h}_{1}=\{(0.6,0.8,0.4)$, $(0.4,0.5,0.9),(0.6,0.2,0.7)\}$ and $\mathfrak{h}_{2}=\{(0.3,0.9,0.5)$, $(0.2,0.4,0.7)\}$ of HT-SFS $\mathbb{T}$ given in Example $1, n=3$; then

$$
\begin{aligned}
S V\left(\mathfrak{h}_{1}\right)= & \frac{1}{\ell_{\mathfrak{h}_{1}}}\left[\left(0.6^{3}-0.4^{3}\right)+\left(0.4^{3}-0.9^{3}\right)\right. \\
& \left.+\left(0.6^{3}-0.7^{3}\right)\right] \\
= & -0.2133 \\
S V\left(\mathfrak{h}_{2}\right)= & \frac{1}{\ell_{\mathfrak{h}_{2}}}\left[\left(0.3^{3}-0.5^{3}\right)+\left(0.2^{3}-0.7^{3}\right)\right] \\
= & -0.2165 .
\end{aligned}
$$

Definition 8 Let $\mathbb{T}_{1}=\left\{\left(x, \mathfrak{h}_{1}(x)\right): x \in \mathfrak{X}\right\}$ and $\mathbb{T}_{2}=$ $\left\{\left(x, \mathfrak{h}_{2}(x)\right): x \in \mathfrak{X}\right\}$ be two HT-SFSs over a common uni-
Fuzzy set

Spherical fuzzy set

Spherical fuzzy number

T-spherical fuzzy set

Initial universe

Membership degree (MD)

Abstinence degree (AD)

Non-membership degree (NMD)

Hesitant T-spherical fuzzy set

Hesitant T-spherical fuzzy element

Score value of hesitant T-spherical fuzzy element $\mathfrak{h}$

Accuracy value of hesitant T-spherical fuzzy element $\mathfrak{h}$

Length of hesitant T-spherical fuzzy element $\mathfrak{h}$

Lower bound of hesitant T-spherical fuzzy element $\mathfrak{h}$

Upper bound of hesitant T-spherical fuzzy element $\mathfrak{h}$

Hesitant T-spherical fuzzy subset

Union operation of HT-SFSs

Intersection operation of HT-SFSs

Complement of HT-SFSs $\mathbb{T}$

Dombi t-norm of $f$ and $g$

Dombi t-conorm of $f$ and $g$

Parameter in Dombi operator

Weight vector with m component

Hesitant T-spherical Dombi fuzzy weighted arithmetic averaging

Hesitant T-spherical Dombi fuzzy weighted geometric averaging

Hesitant T-spherical Dombi fuzzy ordered weighted arithmetic averaging

Hesitant T-spherical Dombi fuzzy ordered weighted geometric averaging verse $\mathfrak{X}$. If, for all $x \in \mathfrak{X} \operatorname{SV}\left(\mathfrak{h}_{1}(x)\right) \leq S V\left(\mathfrak{h}_{2}(x)\right)$, then it is said that $\mathbb{T}_{1}$ is an HT-SF subset of $\mathbb{T}_{2}$, and denoted by $\mathbb{T}_{1} \triangleleft \mathbb{T}_{2}$.

Example 3 Let $\mathbb{T}_{1}$ and $\mathbb{T}_{2}$ be two HT-SFSs over $\mathfrak{X}=$ $\left\{x_{1}, x_{2}, x_{3}\right\}$ for $n=3$ given as follows:

$$
\begin{aligned}
\mathbb{T}_{1}= & \left\{\left(x_{1},\{(0.5,0.4,0.3),(0.6,0.5,0.4),(0.7,0.2,0.6)\}\right),\right. \\
& \left(x_{2},\{(0.6,0.2,0.4),(0.7,0.1,0.6),(0.5,0.5,0.2)\}\right), \\
& \left.\left(x_{3},\{(0.9,0.6,0.1),(0.4,0.4,0.4),(0.6,0.2,0.5)\}\right)\right\} \\
\mathbb{T}_{2}= & \left\{\left(x_{1},\{(0.7,0.6,0.5),(0.7,0.5,0.5),(0.8,0.3,0.6)\}\right),\right. \\
& \left(x_{2},\{(0.8,0.3,0.3),(0.6,0.2,0.2),(0.5,0.7,0.1)\}\right), \\
& \left.\left(x_{3},\{(0.7,0.3,0.3),(0.9,0.2,0.2),(0.5,0.1,0.3)\}\right)\right\} .
\end{aligned}
$$

Then, using Eq. (1), for $x_{i} \in \mathfrak{X}(i=1,2,3)$, SVs of HT-SFEs are obtained as follows:

From the table, it is clear that $\mathbb{T}_{1} \triangleleft \mathbb{T}_{2}$. 


\begin{tabular}{llll}
\hline & $x_{1}$ & $x_{2}$ & $x_{3}$ \\
\hline $\operatorname{SV}\left(\mathfrak{h}_{1}\right)$ & 0.126 & 0.132 & 0.273 \\
$\operatorname{SV}\left(\mathfrak{h}_{2}\right)$ & 0.244 & 0.270 & 0.378 \\
\hline
\end{tabular}

\section{Set-theoretical operations of HT-SFSs}

In this section union, intersection and complement of an HTSFS are defined with their examples.

Definition 9 Let $\mathfrak{h}=\left\{\left(s_{t}, i_{t}, d_{t}\right): 1 \leq t \leq \ell_{\mathfrak{h}}\right\}$ be a T-SFE over $\mathfrak{X}$. Then, lower and upper bounds of $\mathfrak{h}$ are defined as follows:

$$
\begin{aligned}
\mathfrak{h}^{-} & =\min _{t}\left(s_{t}^{n}-d_{t}^{n}\right) \\
\mathfrak{h}^{+} & =\max _{t}\left(s_{t}^{n}-d_{t}^{n}\right),
\end{aligned}
$$

respectively.

The following example can be given to explain lower and upper bound of $\mathfrak{h}$.

Let $\mathfrak{h}=\{(0.5,0.4,0.3),(0.6,0.5,0.4),(0.7,0.2,0.5)\}$ be an HT-FS element, $n=3, l_{\mathfrak{h}}=3$

$$
\begin{aligned}
\mathfrak{h}^{-} & =\min \left\{\left(0.5^{3}-0.3^{3}\right),\left(0.6^{3}-0.4^{3}\right),\left(0.7^{3}-0.5^{3}\right)\right\} \\
& =\min \{0.116,0.152,0.218\} \\
& =0.116 \\
\mathfrak{h}^{+} & =\max \{0.116,0.152,0.218\} \\
& =0.218 .
\end{aligned}
$$

Definition 10 Let $\mathbb{T}_{1}$ and $\mathbb{T}_{2}$ be two HT-SFSs over $\mathfrak{X}$ and let $\mathfrak{h}_{1}$ and $\mathfrak{h}_{2}$ be HT-SFEs of $\mathbb{T}_{1}$ and $\mathbb{T}_{2}$ for all $x \in \mathfrak{X}$. Then, based on HT-SFEs, set-theoretical operations between $\mathbb{T}_{1}$ and $\mathbb{T}_{2}$ are defined as follows:

1. Union:

$$
\begin{aligned}
& \mathbb{T}_{1} \uplus \mathbb{T}_{2}=\bigcup_{x \in \mathfrak{X}}\left\{\left(\begin{array}{l}
\bigcup, \begin{array}{l}
\left.\bigcup_{\left.\left(s_{1}, i_{1}, d_{1}\right) \in i_{k}, d_{k}\right)}\right) \\
\left(s_{2}, i_{2}, d_{2}\right) \in \mathfrak{h}_{2}
\end{array} \\
\mathfrak{h}_{2}
\end{array}\right.\right. \\
& \left.s_{k}^{n}-d_{k}^{n}=\max \left\{s_{1}^{n}-d_{1}^{n}, s_{2}^{n}-d_{2}^{n}\right\}, k=1,2\right\} \\
& \{\}
\end{aligned}
$$

2. Intersection:

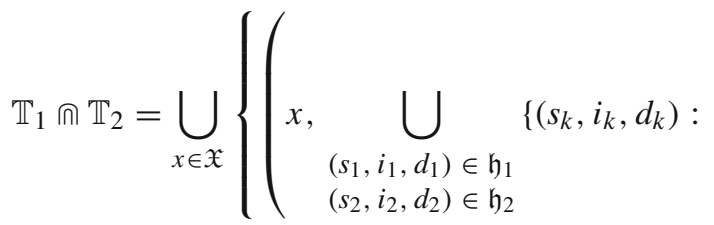

$$
\begin{aligned}
& \left.\left.\left.s_{k}^{n}-d_{k}^{n}=\min \left\{s_{1}^{n}-d_{1}^{n}, s_{2}^{n}-d_{2}^{n}\right\}, k=1,2\right\}\right) \cdot\right\} \\
& \mathbb{T}_{1}^{c}=\bigcup_{x \in \mathfrak{X}}\left\{\left(x, \underset{\left(s_{1}, i_{1}, d_{1}\right) \in \mathfrak{h}_{1}}{\bigcup}\left\{\left(d_{1}, i_{1}, s_{1}\right)\right\}\right)\right\} .
\end{aligned}
$$

Example 4 Let $\mathbb{T}_{1}$ and $\mathbb{T}_{2}$ be two HT-SFSs over $\mathfrak{X}=$ $\left\{x_{1}, x_{2}, x_{3}\right\}$ for $n=4$ given as follows:

$$
\begin{aligned}
\mathbb{T}_{1}= & \left\{\left(x_{1},\{(0.5,0.7,0.9),(0.3,0.2,0.7),(0.6,0.5,0.4)\}\right),\right. \\
& \left(x_{2},\{(0.4,0.6,0.3),\right. \\
& \left.(0.2,0.5,0.9)\}),\left(x_{3},\{(0.8,0.4,0.3)\}\right)\right\} \\
\mathbb{T}_{2}= & \left\{\left(x_{1},\{(0.4,0.8,0.5),(0.5,0.7,0.6)\}\right),\right. \\
& \left(x_{2},\{(0.2,0.7,0.6),(0.4,0.3,0.8)\}\right), \\
& \left.\left(x_{3},\{(0.5,0.4,0.8),(0.6,0.7,0.2),(0.9,0.2,0.4)\}\right)\right\} .
\end{aligned}
$$

Then, using Definition 10, union, intersection. and complement of HT-SFEs are $\mathbb{T}_{1}$ and $\mathbb{T}_{2}$ are obtained as follows:

$$
\begin{aligned}
\mathbb{T}_{1} \cup & \mathbb{T}_{2} \\
= & \left\{\left(x_{1},\{(0.4,0.8,0.5),(0.5,0.7,0.6),(0.6,0.5,0.4)\}\right),\right. \\
& \left(x_{2},\{(0.4,0.6,0.3),(0.2,0.7,0.6),(0.4,0.3,0.8)\}\right), \\
& \left.\left(x_{3},\{(0.8,0.4,0.3),(0.9,0.2,0.4)\}\right)\right\}, \\
\mathbb{T}_{1} & \mathbb{T}_{2} \\
= & \left\{\left(x_{1},\{(0.5,0.7,0.9),(0.3,0.2,0.7),(0.4,0.8,0.5),\right.\right. \\
& (0.5,0.7,0.6)\}), \\
& \left(x_{2},\{(0.2,0.7,0.6),(0.4,0.3,0.8),(0.2,0.5,0.9)\}\right), \\
& \left.\left(x_{3},\{(0.5,0.4,0.8),(0.6,0.7,0.2),(0.8,0.4,0.3)\}\right)\right\}, \\
\text { and } &
\end{aligned}
$$

$$
\begin{aligned}
\mathbb{T}_{1}^{c}= & \left\{\left(x_{1},\{(0.9,0.7,0.5),(0.7,0.2,0.3),(0.4,0.5,0.6)\}\right),\right. \\
& \left(x_{2},\{(0.3,0.6,0.4),\right. \\
& \left.(0.9,0.5,0.2)\}),\left(x_{3},\{(0.3,0.4,0.8)\}\right)\right\},
\end{aligned}
$$

respectively. 


\section{Hesitant T-spherical Dombi fuzzy aggrega- tion operators}

Aggregation operators are an important tool for obtaining a single value from many values. In this section, first, we define Dombi operators for two HT-SFEs based on Dombi t-norm and t-conorm. An HT-SFE is a collection of T-SFEs which have three components called MD, AD, and NMD. In summation $(\oplus)$ operation between HT-SFEs, we use Dombi $\mathrm{t}$-conorm for MDs, Dombi t-norm for AD, and Dombi t-norm for NMD. In product $(\otimes)$ operation between HT-SFEs, we use Dombi t-conorm for MDs, Dombi t-norm for AD, and Dombi t-norm for NMD. Then, we define hesitant T-spherical Dombi fuzzy weighted arithmetic averaging operators and hesitant T-spherical Dombi fuzzy weighted geometric averaging operators as a generalization of the Dombi operators for two HT-SFEs. In these operations, only HT-SFEs are weighted and ignored the importance of the ordered position of HT-SFEs. This is a drawback, and to avoid this drawback, we introduce hesitant T-spherical Dombi fuzzy ordered weighted arithmetic (geometric) averaging operators. In these operators, we consider both the weights of the elements and the importance degrees of the hesitant fuzzy elements according to the score functions. Namely, First, we sort the HT-SFEs according to their score values using the score function we defined, then upon this ordering, we discard the weight vectors given at the beginning without changing the order. The basic idea related to the ordered weighted averaging (OWA) is presented in [73].

\section{Dombi t-norm and t-conorm}

Dombi product and Dombi sum which are specific types of triangular norms and conorms given in [74] as follows:

Definition 11 [74] Let $f$ and $g$ be two real numbers in the interval $[0,1]$. Then, Dombi t-norm is given by

$$
f \otimes g=\frac{1}{1+\left(\left(\frac{1-f}{f}\right)^{\gamma}+\left(\frac{1-g}{g}\right)^{\gamma}\right)^{\frac{1}{\gamma}}}, \quad \gamma>0 .
$$

Dombi t-conorm is given by

$$
f \oplus g=1-\frac{1}{1+\left(\left(\frac{1-f}{f}\right)^{-\gamma}+\left(\frac{1-g}{g}\right)^{-\gamma}\right)^{\frac{1}{\gamma}}}, \quad \gamma>0,
$$

respectively.

\section{Dombi operations of HT-SFEs}

In this subsection, we define some Dombi operations between HT-SFEs.

Definition 12 Let $\mathfrak{h}_{1}=\left\{\left(s_{1 t}, i_{1 t}, d_{1 t}\right): 1 \leq t \leq \ell_{\mathfrak{h}_{1}}\right\}$ and $\mathfrak{h}_{2}=\left\{\left(s_{2 r}, i_{2 r}, d_{2 r}\right): 1 \leq r \leq \ell_{\mathfrak{h}_{2}}\right\}$ be two HT-SFEs and $\gamma>0$, and the Dombi operations for HT-SF elements are defined as follows:

1. $\mathfrak{h}_{1} \oplus \mathfrak{h}_{2}=\bigcup_{\substack{\left(s_{1 t}, i_{1 t}, d_{1 t}\right) \in \mathfrak{h}_{1} \\\left(s_{2 r}, i_{2 r}, d_{2 r}\right) \in \mathfrak{h}_{2}}}$

$$
\begin{aligned}
& \left\{\sqrt[n]{1-\frac{1}{1+\left\{\left(\frac{s_{1 t}^{n}}{1-s_{1 t}^{n}}\right)^{\gamma}+\left(\frac{s_{2 r}^{n}}{1-s_{2 r}^{n}}\right)^{\gamma}\right\}^{\frac{1}{\gamma}}}}\right. \\
& \sqrt[n]{\frac{1}{1+\left\{\left(\frac{1-i_{1 t}^{n}}{i_{1 t}^{n}}\right)^{\gamma}+\left(\frac{1-i_{2 r}^{n}}{i_{2 r}^{n}}\right)^{\gamma}\right\}^{\frac{1}{\gamma}}}}, \\
& \left.\left.\sqrt[n]{\frac{1}{1+\left\{\left(\frac{1-d_{1 t}^{n}}{d_{1 t}^{n}}\right)^{\gamma}+\left(\frac{1-d_{2 r}^{n}}{d_{2 r}^{n}}\right)^{\gamma}\right\}^{\frac{1}{\gamma}}}}\right)\right\}
\end{aligned}
$$

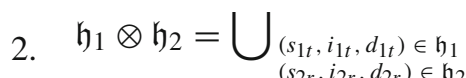

$\left\{\sqrt[n]{\frac{1}{1+\left\{\left(\frac{1-s_{1 t}^{n}}{s_{1 t}^{n}}\right)^{\gamma}+\left(\frac{1-s_{2 r}^{n}}{s_{2 r}^{n}}\right)^{\gamma}\right\}^{\frac{1}{\gamma}}}}\right.$,

$$
\begin{aligned}
& \sqrt[n]{\frac{1}{1+\left\{\left(\frac{1-i_{1 t}^{n}}{i_{1 t}^{n}}\right)^{\gamma}+\left(\frac{1-i_{2 r}^{n}}{i_{2 r}^{n}}\right)^{\gamma}\right\}^{\frac{1}{\gamma}}},} \\
& \sqrt[n]{\left.\left.1-\frac{1}{1+\left\{\left(\frac{d_{1 t}^{n}}{1-d_{1 t}^{n}}\right)^{\gamma}+\left(\frac{d_{2 r}^{n}}{1-d_{2 r}^{n}}\right)^{\gamma}\right\}^{\frac{1}{\gamma}}}\right)\right\}}
\end{aligned}
$$

3.

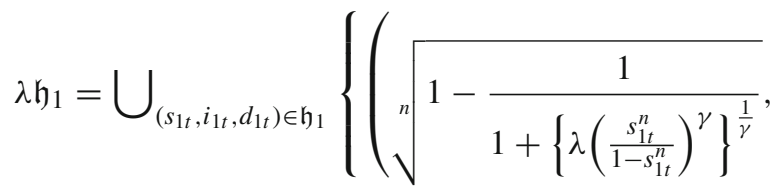

$$
\begin{aligned}
& \sqrt[n]{\frac{1}{1+\left\{\lambda\left(\frac{1-i_{1 t}^{n}}{i_{1 t}^{n}}\right)^{\gamma}\right\}^{\frac{1}{\gamma}}}}, \\
& \sqrt[n]{\left.\left.\frac{1}{1+\left\{\lambda\left(\frac{1-d_{1 t}^{n}}{d_{1 t}^{n}}\right)^{\gamma}\right\}^{\frac{1}{\gamma}}}\right)\right\}}
\end{aligned}
$$


4.

$$
\begin{aligned}
& \mathfrak{h}_{1}^{\lambda}=\bigcup_{\left(s_{1 t}, i_{1 t}, d_{1 t}\right) \in \mathfrak{h}_{1}}\left\{\left(\sqrt[n]{\frac{1}{1+\left\{\lambda\left(\frac{1-s_{1 t}^{n}}{s_{1 t}^{n}}\right)^{\gamma}\right\}^{\frac{1}{\gamma}}}},\right.\right. \\
& \sqrt[n]{\frac{1}{1+\left\{\lambda\left(\frac{1-i_{1 t}^{n}}{i_{1 t}^{n}}\right)^{\gamma}\right\}^{\frac{1}{\gamma}}}}, \\
& \sqrt[n]{\left.\left.1-\frac{1}{1+\left\{\lambda\left(\frac{d_{1 t}^{n}}{1-d_{1 t}^{n}}\right)^{\gamma}\right\}^{\frac{1}{\gamma}}}\right) \cdot\right\}}
\end{aligned}
$$

Example 5 Let us consider $\mathfrak{h}\left(x_{1}\right)=\mathfrak{h}_{1}=\{(0.6,0.8,0.4)$, $(0.4,0.5,0.9),(0.6,0.2,0.7)\}$ and $\mathfrak{h}\left(x_{2}\right)=\mathfrak{h}_{2}=\{(0.3,0.9$, $0.5),(0.2,0.4,0.7)\}$ in Example 1. For $n=3, \gamma=1$, and $\lambda=2$

$$
\begin{aligned}
& \mathfrak{h}_{1} \oplus \mathfrak{h}_{2} \\
&=\{(0.6151,0.7549,0.3536), \\
&(0.6045,0.3922,0.3849),(0.4443,0.4925,0.4925), \\
&(0.4141,0.3536,0.6726),(0.6151,0.1998,0.4655), \\
&(0.6045,0.1928,0.5915)\}
\end{aligned}
$$

$\mathfrak{h}_{1} \otimes \mathfrak{h}_{2}$

$=\{(0.2908,0.7549,0.5587)$,

(0.1981, 0.3922, 0.7187), (0.2685, 0.4925, 0.9041),

$(0.1928,0.3536,0.9136),(0.2908,0.1998,0.7364)$,

$(0.1981,0.1928,0.7994)\}$

$2 \mathfrak{h}_{1}=\{(0.7082,0.7007,0.3209)$,

$(0.4937,0.4055,0.8309),(0.7082,0.1590,0.5915)\}$

$\mathfrak{h}_{1}^{2}=\{(0.4947,0.7007,0.4937)$,

$(0.3209,0.4055,0.9448),(0.4947,0.1590,0.7994)\}$.

\section{Hesitant T-spherical Dombi fuzzy weighted arithmetic averaging operator}

Definition 13 Let $\mathcal{H}^{m}=\left\{\mathfrak{h}_{k}=\left\{\left(s_{k j}, i_{k j}, d_{k j}\right): 1 \leq\right.\right.$ $\left.j \leq \ell_{\mathfrak{h}_{k}}, k=1,2, \ldots, m\right\}$ be an $m$ dimensional collection of HT-SFEs. A hesitant T-spherical Dombi fuzzy weighted averaging (HTSDFWAA) operator is defined by a function HTSDFWAA: $\mathcal{H}^{m} \rightarrow \mathcal{H}$ as follows:

$$
\begin{aligned}
& \operatorname{HTSDFWAA}\left(\mathfrak{h}_{1}, \mathfrak{h}_{2}, \mathfrak{h}_{3}, \ldots, \mathfrak{h}_{m}\right) \\
& =\bigoplus_{z=1}^{m}\left(w_{z} \mathfrak{h}_{z}\right) \\
& =\left(w_{1} \mathfrak{h}_{1}\right) \oplus\left(w_{2} \mathfrak{h}_{2}\right) \oplus \cdots \oplus\left(w_{m} \mathfrak{h}_{m}\right),
\end{aligned}
$$

where $w_{z}$ is weight of $\mathfrak{h}_{z}(z=1,2, \ldots, m), 0 \leq w_{z} \leq 1$ and $\sum_{z=1}^{m} w_{z}=1$.
We get the following theorem that follows the Dombi operations on HT-SFEs.

Theorem 1 Let $\mathfrak{h}_{k} \in \mathcal{H}^{m}$. Then

$$
\begin{aligned}
& H T S D F W A A\left(\mathfrak{h}_{1}, \mathfrak{h}_{2}, \ldots, \mathfrak{h}_{m}\right) \\
& =\bigoplus_{z=1}^{m}\left(w_{z} \mathfrak{h}_{z}\right)
\end{aligned}
$$

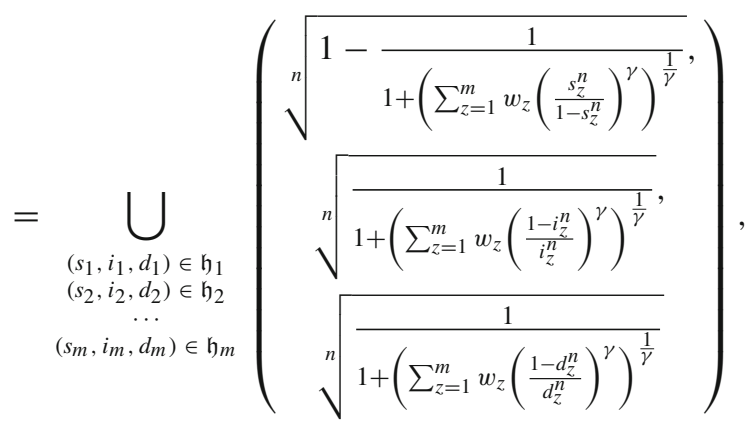

where $\omega=\left(w_{1}, w_{2}, \ldots, w_{m}\right)$ be the $m$ weight vector of $\mathfrak{h}_{k}(k=1,2, \ldots, m)$, such that $\omega_{k}>0$ and $\sum_{k=1}^{m} w_{k}=1$.

Proof The theorem can be proved by the method of mathematical induction as follows:

(i) When $m=2$, based on Dombi operations on HT-SFEs, we obtain the following results:

$$
\begin{aligned}
& w_{1} \mathfrak{h}_{1}=\bigcup_{\left(s_{1}, i_{1}, d_{1}\right) \in \mathfrak{h}_{1}}\left(\begin{array}{l}
\sqrt[n]{\frac{1-\frac{1}{1+\left(w_{1}\left(\frac{s_{1}^{n}}{1-s_{1}^{n}}\right)^{\gamma}\right)^{\frac{1}{\gamma}}}}{1+\left(w_{1}\left(\frac{1-i_{1}^{n}}{i_{1}^{n}}\right)^{\gamma}\right)^{\frac{1}{\gamma}}}}, \\
\sqrt[n]{\frac{1}{1+\left(w_{1}\left(\frac{1-d_{1}^{n}}{d_{1}^{n}}\right)^{\gamma}\right)^{\frac{1}{\gamma}}}}
\end{array}\right) \\
& w_{2} \mathfrak{h}_{2}=\bigcup_{\left(s_{2}, i_{2}, d_{2}\right) \in \mathfrak{h}_{2}}\left(\begin{array}{l}
\sqrt[n]{\sqrt[n]{\frac{1-\frac{1}{1+\left(w_{2}\left(\frac{s_{2}^{n}}{1-s_{2}^{n}}\right)^{\gamma}\right)^{\frac{1}{\gamma}}}}{1+\left(w_{2}\left(\frac{1-i_{2}^{n}}{i_{2}^{n}}\right)^{\gamma}\right)^{\frac{1}{\gamma}}},}} \\
\sqrt[n]{\frac{1}{1+\left(w_{2}\left(\frac{1-d_{2}^{n}}{d_{2}^{n}}\right)^{\gamma}\right)^{\frac{1}{\gamma}}}}
\end{array}\right)
\end{aligned}
$$

$w_{1} \mathfrak{h}_{1} \oplus w_{2} \mathfrak{h}_{2}$ 


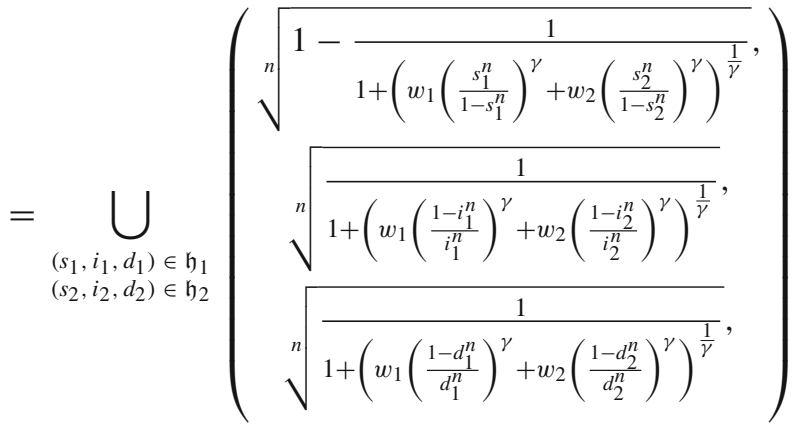

$w_{1} \mathfrak{h}_{1} \oplus w_{2} \mathfrak{h}_{2}$

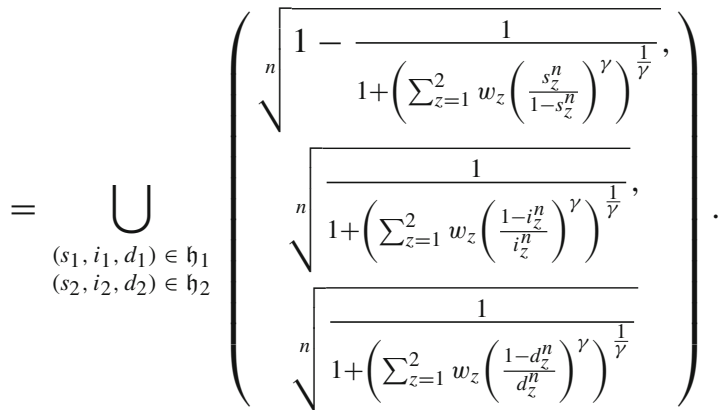

Then, the theorem holds for $m=2$

(ii) Suppose the theorem holds when $z=k$ that is

$$
\begin{aligned}
& \oplus_{z=1}^{k}\left(w_{z} \mathfrak{h}_{z}\right) \\
& =\bigcup \\
& \left(s_{1}, i_{1}, d_{1}\right) \in \mathfrak{h}_{1} \\
& \left(\begin{array}{c}
\sqrt[n]{1-\frac{1}{1+\left(\sum_{z=1}^{k} w_{z}\left(\frac{s_{z}^{n}}{1-s_{z}^{n}}\right)^{\gamma}\right)^{\frac{1}{\gamma}}}}, \\
\sqrt[n]{\frac{1}{1+\left(\sum_{z=1}^{k} w_{z}\left(\frac{1-i_{z}^{n}}{i_{z}^{n}}\right)^{\gamma}\right)^{\frac{1}{\gamma}}},} \\
\sqrt[n]{\frac{1}{1+\left(\sum_{z=1}^{k} w_{z}\left(\frac{1-d_{z}^{n}}{d_{z}^{n}}\right)^{\gamma}\right)^{\frac{1}{\gamma}}}}
\end{array}\right)
\end{aligned}
$$

When $z=k+1$

$$
\begin{aligned}
& \oplus_{z=1}^{k}\left(w_{z} \mathfrak{h}_{z}\right) \oplus w_{k+1} \mathfrak{h}_{k+1}
\end{aligned}
$$

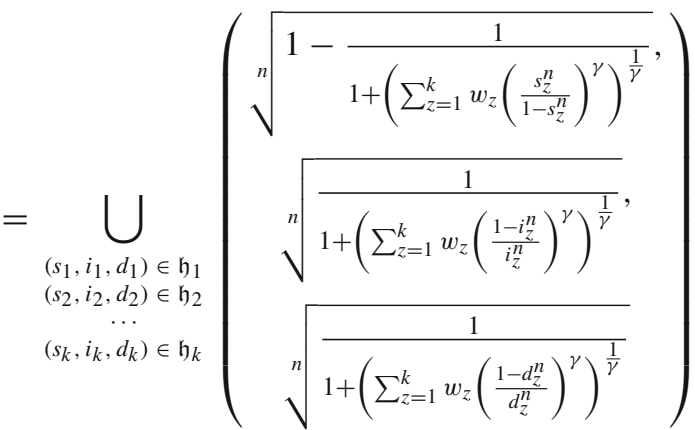

$$
\begin{aligned}
& \bigoplus w_{k+1} \mathfrak{h}_{k+1} \\
& \oplus_{z=1}^{k}\left(w_{z} \mathfrak{h}_{z}\right) \oplus w_{k+1} \mathfrak{h}_{k+1}
\end{aligned}
$$
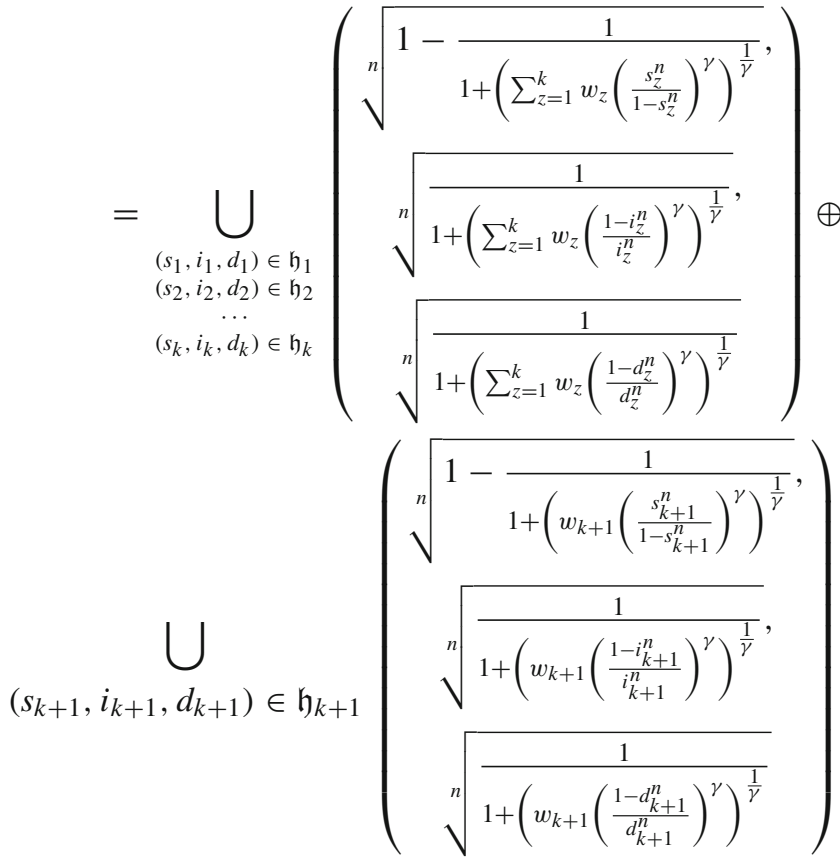

$\oplus_{z=1}^{k+1}\left(w_{z} \mathfrak{h}_{z}\right)$

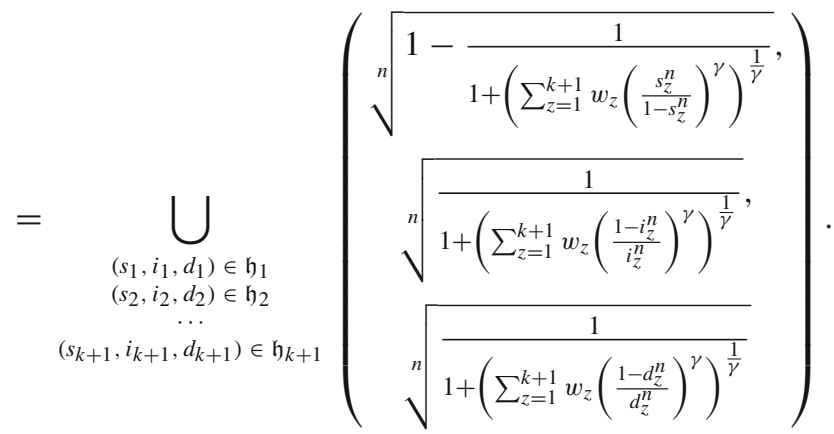

Then, the theorem holds for $z=k+1$. Hence, the theorem is proved for all $z \in \mathbb{N}$.

Example 6 Let us consider $\mathfrak{h}_{1}=\{(0.6,0.8,0.4),(0.4,0.5$, $0.9),(0.6,0.2,0.7)\}, \mathfrak{h}_{2}=\{(0.3,0.9,0.5),(0.2,0.4,0.7)\}$, and $\mathfrak{h}_{3}=\{(0.5,0.4,0.3)\}$ for $n=3$. When $\gamma=1$, with weighted vector $\omega=(0.5,0.3,0.2)$, we get

$$
\begin{aligned}
& \operatorname{HTSDFWAA}\left(\mathfrak{h}_{1}, \mathfrak{h}_{2}, \mathfrak{h}_{3}\right)=\oplus_{z=1}^{3}\left(w_{z} \mathfrak{h}_{z}\right)
\end{aligned}
$$

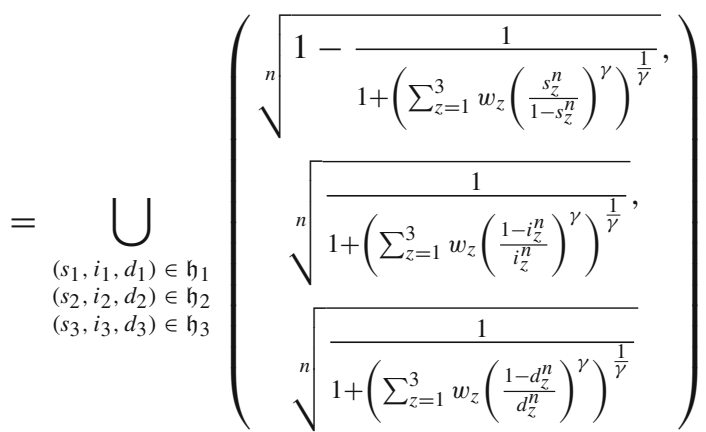


$\operatorname{HTSDFWAA}\left(\mathfrak{h}_{1}, \mathfrak{h}_{2}, \mathfrak{h}_{3}\right)$

$=\{(0.5298,0.6051,0.3843),(0.5246,0.4846,0.3961)$, $(0.4049,0.5100,0.4568),(0.3941,0.4391,0.4813)$,

$(0.5298,0.2474,0.4461),(0.5246,0.2423,0.4683)\}$.

Theorem 2 (Idempotency) Let $\mathfrak{h}_{k}(k=1,2, \ldots, m)$ be a number of HT-SFNs. Then, $\mathfrak{h}_{k}=\left(s_{k}, i_{k}, d_{k}\right)(k=$ $1,2, \ldots, m)$ be a number of HT-SFEs are all equal, i.e., $\mathfrak{h}_{k}=\mathfrak{h}$ for all $k$, then HTSDFWAA $\left(\mathfrak{h}_{1}, \mathfrak{h}_{2}, \ldots, \mathfrak{h}_{m}\right)=\mathfrak{h}$.

\section{Hesitant T-spherical Dombi fuzzy weighted geometric averaging (HTSDFWGA) operator}

Definition 14 Let $\mathcal{H}^{m}=\left\{\mathfrak{h}_{k}=\left\{\left(s_{k j}, i_{k j}, d_{k j}\right): 1 \leq j \leq\right.\right.$ $\left.\ell_{\mathfrak{h}_{k}}, k=1,2, \ldots, m\right\}$ be an m-dimensional collection of HTSFEs. An HTSDFWGA operator is defined by a function HTSDFWGA: $\mathcal{H}^{m} \rightarrow \mathcal{H}$ as follows:

$$
\begin{aligned}
& \operatorname{HTSDFWGA}\left(\mathfrak{h}_{1}, \mathfrak{h}_{2}, \mathfrak{h}_{3}, \ldots, \mathfrak{h}_{m}\right) \\
& =\bigotimes_{z=1}^{m}\left(\mathfrak{h}_{z}^{w_{z}}\right) \\
& =\left(\mathfrak{h}_{1}^{w_{1}}\right) \otimes\left(\mathfrak{h}_{2}^{w_{2}}\right) \otimes \cdots \otimes\left(\mathfrak{h}_{m}^{w_{m}}\right),
\end{aligned}
$$

where $w_{z}$ is weight of $\mathfrak{h}_{z}(z=1,2, \ldots, m), 0 \leq w_{z} \leq 1$ and $\sum_{z=1}^{m} w_{z}=1$.

Theorem 3 Let $\mathfrak{h}_{k} \in \mathcal{H}^{m}$. Then

$$
\begin{aligned}
& \operatorname{HTSDFWGA}\left(\mathfrak{h}_{1}, \mathfrak{h}_{2}, \ldots, \mathfrak{h}_{m}\right)=\bigotimes_{z=1}^{m}\left(\mathfrak{h}_{z}^{w_{z}}\right)
\end{aligned}
$$

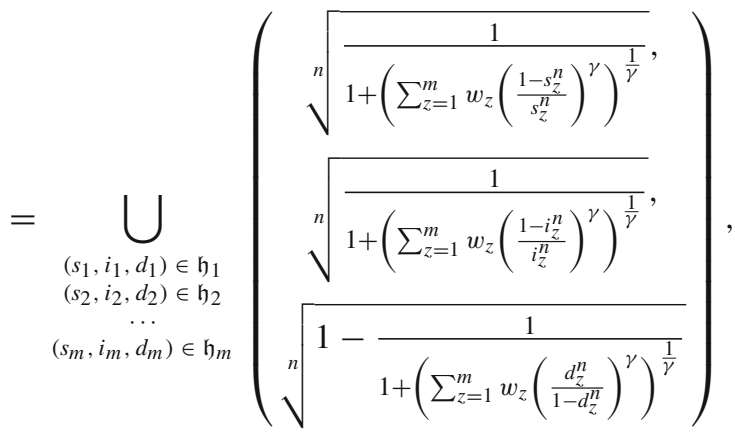

where $\omega=\left(w 1, w_{2}, \ldots, w_{m}\right)$ be the $m$ weighted vector of $\mathfrak{h}_{k}(k=1,2, \ldots, m)$, such that $w_{k}>0$ and $\sum_{k=1}^{m} w_{k}=1$.

Proof The theorem can be proved by the mathematical induction as follows:

(i) When $m=2$, we have

$$
\mathfrak{h}_{1}^{w_{1}}=
$$

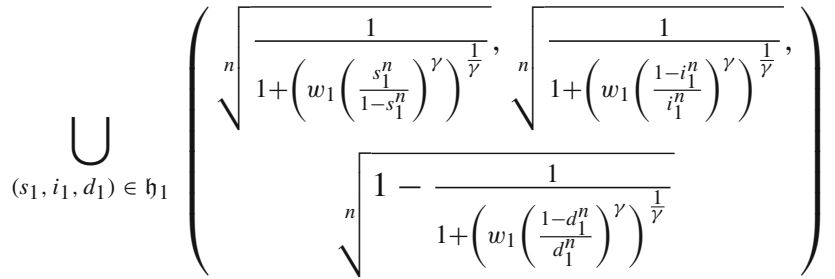

$\mathfrak{h}_{2}^{w_{2}}=$

$\bigcup_{\left(s_{2}, i_{2}, d_{2}\right) \in \mathfrak{h}_{2}}\left(\frac{\sqrt[n]{\frac{1}{1+\left(w_{2}\left(\frac{s_{2}^{n}}{1-s_{2}^{n}}\right)^{\gamma}\right)^{\frac{1}{\gamma}}}, \sqrt[n]{\frac{1}{1+\left(w_{2}\left(\frac{1-i_{2}^{n}}{i_{2}^{n}}\right)^{\gamma}\right)^{\frac{1}{\gamma}}}},}}{\sqrt[n]{1-\frac{1}{1+\left(w_{2}\left(\frac{1-d_{2}^{n}}{d_{2}^{n}}\right)^{\gamma}\right)^{\frac{1}{\gamma}}}}}\right)$

and

$$
=\bigcup_{\substack{\left(s_{1}, i_{1}, d_{1}\right) \in \mathfrak{h}_{1} \\
\left(s_{2}, i_{2}, d_{2}\right) \in \mathfrak{h}_{2}}}\left(\begin{array}{c}
\mathfrak{h}_{1}^{w_{1}} \otimes \mathfrak{h}_{2}^{w_{2}} \\
\sqrt[n]{\frac{\sqrt[n]{1+\left(w_{1}\left(\frac{s_{1}^{n}}{1-s_{1}^{n}}\right)^{\gamma}+w_{2}\left(\frac{s_{2}^{n}}{1-s_{2}^{n}}\right)^{\gamma}\right)^{\frac{1}{\gamma}}}}{1+\left(w_{1}\left(\frac{1-i_{1}^{n}}{i_{1}^{n}}\right)^{\gamma}+w_{2}\left(\frac{1-i_{2}^{n}}{i_{2}^{n}}\right)^{\gamma}\right)^{\frac{1}{\gamma}}},} \\
\sqrt[n]{1-\frac{1}{1+\left(w_{1}\left(\frac{1-d_{1}^{n}}{d_{1}^{n}}\right)^{\gamma}+w_{2}\left(\frac{1-d_{2}^{n}}{d_{2}^{n}}\right)^{\gamma}\right)^{\frac{1}{\gamma}}}},
\end{array}\right)
$$

$\mathfrak{h}_{1}^{w_{1}} \otimes \mathfrak{h}_{2}^{w_{2}}$

$$
=\bigcup_{\substack{\left(s_{1}, i_{1}, d_{1}\right) \in \mathfrak{h}_{1} \\
\left(s_{2}, i_{2}, d_{2}\right) \in \mathfrak{h}_{2}}}\left(\begin{array}{l}
\sqrt[n]{\frac{1}{1+\left(\sum_{z=1}^{2} w_{z}\left(\frac{s_{z}^{n}}{1-s_{z}^{n}}\right)^{\gamma}\right)^{\frac{1}{\gamma}}},} \\
\sqrt[n]{1-\frac{1}{1+\left(\sum_{z=1}^{2} w_{z}\left(\frac{1-i_{z}^{n}}{i_{z}^{n}}\right)^{\gamma}\right)^{\frac{1}{\gamma}}},}
\end{array} .\right.
$$

Then, the theorem holds for $m=2$.

(ii) Suppose that the theorem holds for $z=k$, that is

$$
\otimes_{z=1}^{k}\left(\mathfrak{h}_{z}^{w_{z}}\right)
$$

$$
=\bigcup_{\substack{\left(s_{1}, i_{1}, d_{1}\right) \in \mathfrak{h}_{1} \\\left(s_{2}, i_{2}, d_{2}\right) \in \mathfrak{h}_{2} \\\left(s_{k}, i_{k}, d_{k}\right) \in \mathfrak{h}_{k}}}\left(\sqrt[n]{\sqrt[n]{\frac{1}{1+\left(\sum_{z=1}^{k} w_{z}\left(\frac{s_{z}^{n}}{1-s_{z}^{n}}\right)^{\gamma}\right)^{\frac{1}{\gamma}}},}}\right) .
$$


We prove that equation is true for $z=k+1$

$$
\begin{aligned}
& \otimes_{z=1}^{k}\left(\mathfrak{h}_{z}^{w_{z}}\right) \otimes \mathfrak{h}_{k+1}^{w_{k+1}}
\end{aligned}
$$

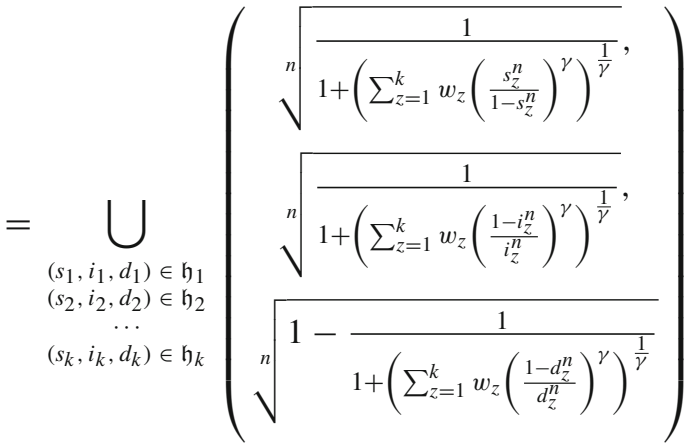

$$
\begin{aligned}
& \otimes \mathfrak{h}_{k+1}^{\omega_{k+1}} \otimes_{z=1}^{k}\left(\mathfrak{h}_{z}^{w_{z}}\right) \otimes \mathfrak{h}_{k+1}^{w_{k+1}}
\end{aligned}
$$

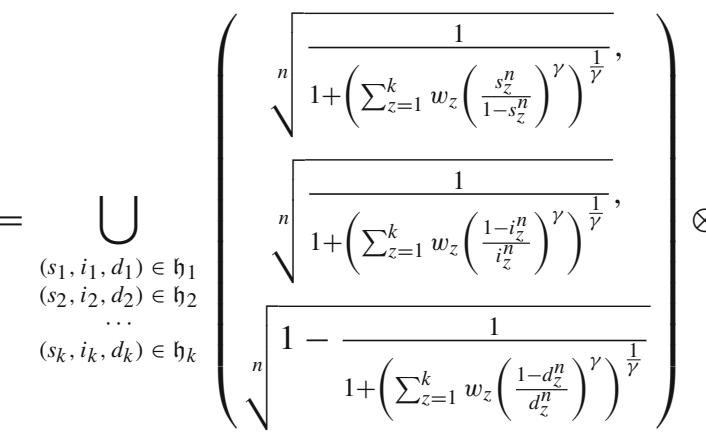

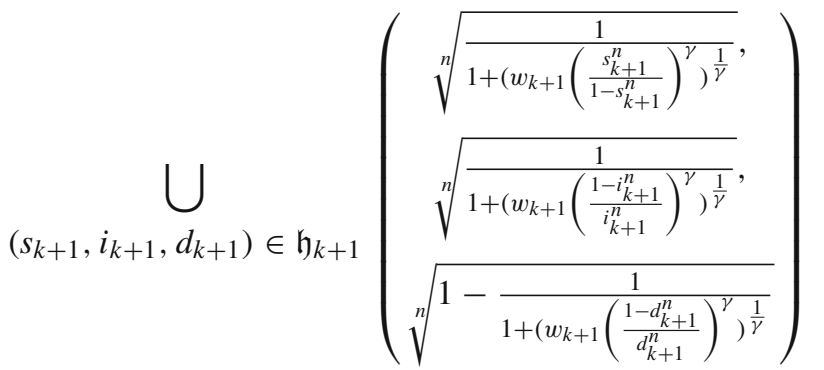

$$
\begin{aligned}
& \otimes_{z=1}^{k+1}\left(\mathfrak{h}_{z}^{w_{z}}\right) \\
& = \\
& \begin{array}{l|l}
\bigcup_{\substack{\left(s_{1}, i_{1}, d_{1}\right) \in \mathfrak{h}_{1} \\
\left(s_{2}, i_{2}, d_{2}\right) \in \mathfrak{h}_{2} \\
\cdots \\
\left(s_{k+1}, i_{k+1}, d_{k+1}\right) \in \mathfrak{h}_{k+1}}} & \sqrt[n]{\sqrt[n]{1-\frac{1}{1+\left(\sum_{z=1}^{k+1} w_{z}\left(\frac{1-i_{z}^{n}}{i_{z}^{n}}\right)^{\gamma}\right)^{\frac{1}{\gamma}}},}} \\
\sqrt[n]{1+\left(\sum_{z=1}^{k+1} w_{z}\left(\frac{1-d_{z}^{n}}{d_{z}^{n}}\right)^{\gamma}\right)^{\frac{1}{\gamma}}}
\end{array}
\end{aligned}
$$

Then, the theorem holds for $z=k+1$. Hence, the proof is completed.

Example 7 Let us consider HT-SFEs given in Example 6. Then, we get

$$
\operatorname{HTSDFWGA}\left(\mathfrak{h}_{1}, \mathfrak{h}_{2}, \mathfrak{h}_{3}\right)=\otimes_{z=1}^{3}\left(\mathfrak{h}_{z}^{w_{z}}\right)
$$

$$
=\bigcup_{\substack{\left(s_{1}, i_{1}, d_{1}\right) \in \mathfrak{h}_{1} \\\left(s_{2}, i_{2}, d_{2}\right) \in \mathfrak{h}_{2} \\\left(s_{3}, i_{3}, d_{3}\right) \in \mathfrak{h}_{3}}} \sqrt[n]{\sqrt[n]{\frac{1}{1+\left(\sum_{z=1}^{3} w_{z}\left(\frac{s_{z}^{n}}{1-s_{z}^{n}}\right)^{\gamma}\right)^{\frac{1}{\gamma}}},}} \sqrt[n]{\sqrt{1-\frac{1}{1+\left(\sum_{z=1}^{3} w_{z}\left(\frac{1-i_{z}^{n}}{i_{z}^{n}}\right)^{\gamma}\right)^{\frac{1}{\gamma}}},}}
$$

$\operatorname{HTSDFWGA}\left(\mathfrak{h}_{1}, \mathfrak{h}_{2}, \mathfrak{h}_{3}\right)$

$=\{(0.4053,0.6051,0.4241),(0.2890,0.4846,0.5475)$,

(0.3652, 0.5100, 0.8350), (0.2773, 0.4391, 0.8440),

$(0.4052,0.2474,0.6183),(0.2890,0.2423,0.6675)\}$.

Theorem 4 (Idempotency) Let $\mathfrak{h}_{k} \in \mathcal{H}^{m}(k=1,2, \ldots, m)$. If $\mathfrak{h}_{k}=\mathfrak{h}$ for $k=1,2, \ldots, m$, then HTSDFWGA $\left(\mathfrak{h}_{1}, \mathfrak{h}_{2}\right.$, $\left.\ldots, \mathfrak{h}_{m}\right)=\mathfrak{h}$.

Proof Straightforward. Therefore, the proof is omitted.

\section{Hesitant T-spherical Dombi fuzzy ordered weighted arithmetic averaging (HTSDFOWAA) operator}

Definition 15 Let $\mathcal{H}^{m}=\left\{\mathfrak{h}_{k}=\left\{\left(s_{k j}, i_{k j}, d_{k j}\right): 1 \leq j \leq\right.\right.$ $\left.\ell_{\mathfrak{h}_{k}}, k=1,2, \ldots, m\right\}$ be an $\mathrm{m}$ dimensional collection of HTSFEs. An HTSDFOWAA operator is defined by a function HTSDFOWAA: $\mathcal{H}^{m} \rightarrow \mathcal{H}$ as follows:

$$
\begin{aligned}
& \operatorname{HTSDFOWAA}\left(\mathfrak{h}_{1}, \mathfrak{h}_{2}, \mathfrak{h}_{3}, \ldots, \mathfrak{h}_{m}\right) \\
& =\bigoplus_{z=1}^{m}\left(w_{z} \mathfrak{h}_{\sigma(z)}\right) \\
& =\left(w_{1} \mathfrak{h}_{\sigma(1)}\right) \oplus\left(w_{2} \mathfrak{h}_{\sigma(2)}\right) \oplus \cdots \oplus\left(w_{m} \mathfrak{h}_{\sigma(m)}\right),
\end{aligned}
$$

where $\mathfrak{h}_{\sigma(z)}$ is the zth largest of $\mathfrak{h}_{z}$ and $w_{z}$ is weighted vector of $\mathfrak{h}_{z}(z=1,2, \ldots, m), 0 \leq w_{z} \leq 1$ and $\sum_{z=1}^{m} w_{z}=1$.

Theorem 5 Let $\mathfrak{h}_{k} \in \mathcal{H}^{m}(k=1,2, \ldots, m)$. Then

$$
\begin{aligned}
& \operatorname{HTSDFOWAA}\left(\mathfrak{h}_{1}, \mathfrak{h}_{2}, \ldots, \mathfrak{h}_{m}\right)=\bigoplus_{z=1}^{m}\left(w_{z} \mathfrak{h}_{\sigma(z)}\right) \\
& =\bigcup \\
& \left(s_{1}, i_{1}, d_{1}\right) \in \mathfrak{h}_{1} \\
& \left(s_{2}, i_{2}, d_{2}\right) \in \mathfrak{h}_{2} \\
& \left(s_{m}, i_{m}, d_{m}\right) \in \mathfrak{h}_{m} \\
& \begin{array}{c}
\sqrt[n]{\sqrt{1-\frac{1}{1+\left(\sum_{z=1}^{m} w_{z}\left(\frac{s_{\sigma(z)}^{n}}{1-s_{\sigma(z)}^{n}}\right)^{\gamma}\right)^{\frac{1}{\gamma}}}},} \\
\sqrt[n]{\frac{1}{1+\left(\sum_{z=1}^{m} w_{z}\left(\frac{\left.\left.1-i_{\sigma(z)}^{n}\right)^{\gamma}\right)^{\frac{1}{\gamma}}}{i_{\sigma(z)}}\right)\right.}} \\
\sqrt[n]{\frac{1}{1+\left(\sum_{z=1}^{m} w_{z}\left(\frac{1-d_{\sigma(z)}^{n}}{d_{\sigma(z)}^{n}}\right)^{\gamma}\right)^{\frac{1}{\gamma}}}}
\end{array},
\end{aligned}
$$


whereh $_{\sigma(z)}$ is the zth largest of $\mathfrak{h}_{z}$ and $\omega=\left(w_{1}, w_{2}, \ldots, w_{m}\right)$ be the $m$ weighted vector of $\mathfrak{h}_{k}(k=1,2, \ldots, m)$, such that $0<w_{k}<1$ and $\sum_{k=1}^{m} w_{k}=1$

Proof The proof is made by similar way to proof of Theorem 1.

\section{Hesitant T-spherical Dombi fuzzy ordered weighted geometric averaging (HTSDFOWGA) operator}

Definition 16 Let $\mathcal{H}^{m}=\left\{\mathfrak{h}_{k}=\left\{\left(s_{k j}, i_{k j}, d_{k j}\right): 1 \leq j \leq\right.\right.$ $\left.\left.\ell_{\mathfrak{h}_{k}}\right\}, k=1,2, \ldots, m\right\}$ be an $\mathrm{m}$ dimensional collection of HT-SFEs. An HTSDFOWGA operator is defined by a function HTSDFOWGA: $\mathcal{H}^{m} \rightarrow \mathcal{H}$ as follows:

$$
\begin{aligned}
& \operatorname{HTSDFOWGA}\left(\mathfrak{h}_{1}, \mathfrak{h}_{2}, \mathfrak{h}_{3}, \ldots, \mathfrak{h}_{m}\right) \\
& =\bigotimes_{z=1}^{m}\left(\mathfrak{h}_{\sigma(z)}^{w_{z}}\right) \\
& =\left(\mathfrak{h}_{1}^{w_{1}}\right) \otimes\left(\mathfrak{h}_{2}^{w_{2}}\right) \otimes \cdots \otimes\left(\mathfrak{h}_{\sigma(m)}^{w_{m}}\right),
\end{aligned}
$$

where $w_{z}$ is weighted vector of $\mathfrak{h}_{z}(z=1,2, \ldots, m), 0 \leq$ $w_{z} \leq 1$ and $\sum_{z=1}^{m} w_{z}=1$.

Theorem 6 Let $\mathfrak{h}_{k} \in \mathcal{H}^{m}$. Then

$$
\begin{aligned}
& \operatorname{HTSDFOWGA}\left(\mathfrak{h}_{\sigma(1)}, \mathfrak{h}_{\sigma(2)}, \ldots, \mathfrak{h}_{m}\right)=\bigotimes_{z=1}^{m}\left(\mathfrak{h}_{\sigma(z)}^{w_{z}}\right)
\end{aligned}
$$

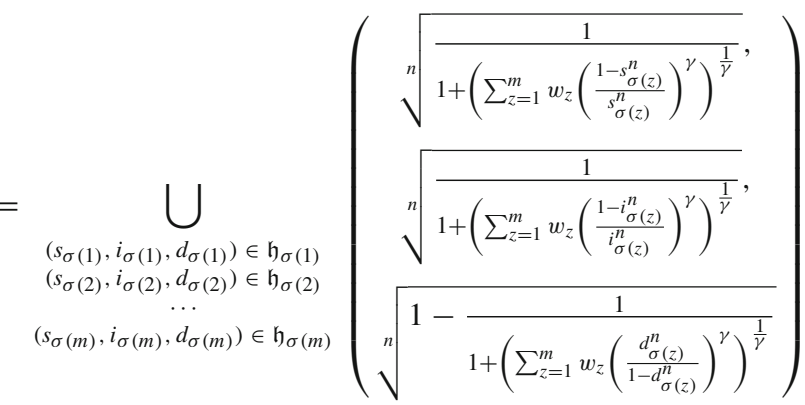

where $\mathfrak{h}_{\sigma(z)}$ is the $z$ th largest of $\mathfrak{h}_{z}$ and $\omega=\left(w_{1}, w_{2}, \ldots\right.$, $\left.w_{m}\right)$ be the $m$ weighted vector of $\mathfrak{h}_{k}(k=1,2, \ldots, m)$, such that $0<w_{k}<1$ and $\sum_{k=1}^{m} w_{k}=1$.

Example 8 Let us consider $\mathfrak{h}_{1}=\{(0.6,0.8,0.4),(0.4,0.5$, $0.9),(0.6,0.2,0.7)\}, \mathfrak{h}_{2}=\{(0.3,0.9,0.5),(0.2,0.4,0.7)\}$ and $\mathfrak{h}_{3}=\{(0.5,0.4,0.3)\}$ for $n=3$. When $\gamma=1$, with weight vector $\omega=(0.5,0.3,0.2)$, we get

$$
\operatorname{HTSDFOWGA}\left(\mathfrak{h}_{\sigma(1)}, \mathfrak{h}_{\sigma(2)}, \mathfrak{h}_{3}\right)=\bigotimes_{z=1}^{3}\left(\mathfrak{h}_{\sigma(z)}^{w_{z}}\right)
$$

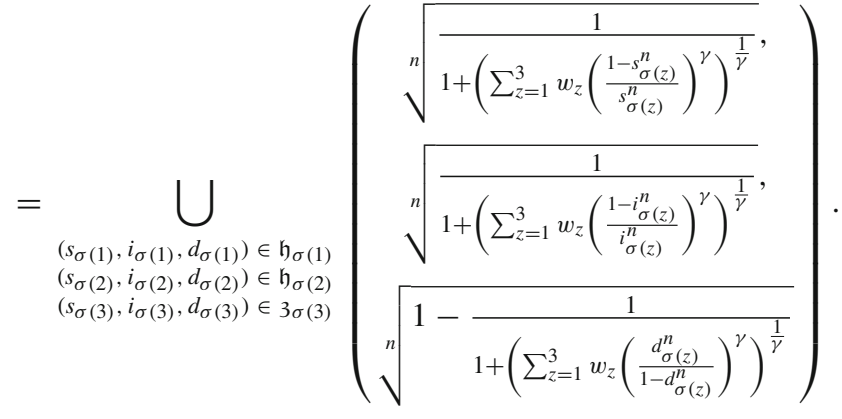

Using Eq. (1), SVs of HT-SFEs are obtained as follows:

$\operatorname{SV}\left(\mathfrak{h}_{1}\right)=-0.2133$

$\operatorname{SV}\left(\mathfrak{h}_{2}\right)=-0.2165$,

$\operatorname{SV}\left(\mathfrak{h}_{3}\right)=0.089$.

Here, $\operatorname{SV}\left(\mathfrak{h}_{3}\right)>\operatorname{SV}\left(\mathfrak{h}_{1}\right)>\operatorname{SV}\left(\mathfrak{h}_{2}\right)$ and

$\mathfrak{h}_{\sigma(1)}=\mathfrak{h}_{3}=\{(0.5,0.4,0.3)\}$

$\mathfrak{h}_{\sigma(2)}=\mathfrak{h}_{1}=\{(0.6,0.8,0.4),(0.4,0.5,0.9),(0.6,0.2,0.7)\}$

$\mathfrak{h}_{\sigma(3)}=\mathfrak{h}_{2}=\{(0.3,0.9,0.5),(0.2,0.4,0.7)\}$.

Then

$\operatorname{HTSDFOWGA}\left(\mathfrak{h}_{1}, \mathfrak{h}_{2}, \mathfrak{h}_{3}\right)=\{(0.4275,0.4867,0.3898)$,

$(0.3961,0.4569,0.7716),(0.4275,0.2799,0.5496)$,

$(0.3205,0.3105,0.4958),(0.3096,0.3051,0.7833)$,

$(0.3205,0.2423,0.5997)\}$.

Theorem 7 (Idempotency property) Let $\mathfrak{h}_{k} \in \mathcal{H}^{m}(k=$ $1,2, \ldots, m)$. If $\mathfrak{h}_{k}=\mathfrak{h}$ for $k=1,2, \ldots, m$, then $\operatorname{HTSDFOWGA}\left(\mathfrak{h}_{1}, \mathfrak{h}_{2}, \ldots, \mathfrak{h}_{m}\right)=\mathfrak{h}$.

Proof Straightforward.

\section{Multiple criteria group decision-making (MCGDM) method under HT-SF information}

In this section, we develop a multiple criteria group decisionmaking method. First, we give frequently used notation in Table 2 for convenience.

Let $\kappa=\left\{\kappa_{1}, \kappa_{2}, \ldots, \kappa_{l}\right\}$ be set of alternatives, $\epsilon=$ $\left\{\epsilon_{1}, \epsilon_{2}, \ldots, \epsilon_{s}\right\}$ be a set of criteria and $\partial=\left\{\partial_{1}, \partial_{2}, \ldots, \partial_{t}\right\}$ be a set of decision-makers. Let us consider $w=\left(w_{1}, w_{2}, \ldots\right.$, $\left.w_{s}\right)$, such that $w_{j} \in(0,1]$ and $\sum_{j=1}^{s} w_{j}=1$ as the weight vector of the criteria which is determined by decision-makers. The steps of the MCGDM method are given as follows:

Step 1: The evaluation of the alternative $\kappa_{i}$ according to criteria $\epsilon_{j}$ performed by decision-makers $\partial_{y}(y=$ 
Table 2 Frequently used notations in "Multiple criteria group decision-making (MCGDM) method under HT-SF information" and other

\begin{tabular}{ll}
\hline$\kappa=\left\{\kappa_{1}, \kappa_{2}, \ldots, \kappa_{l}\right\}$ & Set of alternatives \\
$\epsilon=\left\{\epsilon_{1}, \epsilon_{2}, \ldots, \epsilon_{s}\right\}$ & Set of criteria \\
$\partial=\left\{\partial_{1}, \partial_{2}, \ldots, \partial_{t}\right\}$ & Set of decision-makers \\
$D_{\kappa_{i}}$ & For alternative $\kappa_{i}$ decision matrix \\
$\operatorname{HTSF}_{i}$ & Collection of $i$ column elements of $D_{\kappa_{i}}$ matrix \\
$\zeta_{y j}$ & Element of $D_{\kappa_{i}}$ corresponding to $y$ row and $j$ column \\
$\mathfrak{A}_{i}$ & HT-SFE corresponding to alternative $\kappa_{i}$ \\
\hline
\end{tabular}

$1,2, \ldots, t)$ can be written as $\zeta_{y j}(i=1,2, \ldots, l ; j=$ $1,2, \ldots, s ; y=1,2, \ldots, t)$. Hence, HT-SF-decision matrix $D M_{\kappa_{i}}=\left[\zeta_{y j}\right]_{t \times s}$ can be constructed as follows:

$D_{\kappa_{i}}=\left[\zeta_{y j}\right]_{t \times s}=\left(\begin{array}{cccc}\zeta_{11} & \zeta_{12} & \ldots & \zeta_{1 s} \\ \zeta_{21} & \zeta_{22} & \ldots & \zeta_{2 s} \\ \vdots & \vdots & \ldots & \vdots \\ \zeta_{t 1} & \zeta_{t 2} & \ldots & \zeta_{t s}\end{array}\right)$

Step 2: For all $i=1,2, \ldots, l$, HT-SFS denoted by $\mathrm{HTSF}_{i}$ is obtained as follows:

$H T S F_{i}=\left\{\left(\epsilon_{j}, \mathfrak{h}_{\epsilon_{j}}\right): j=1,2, \ldots, s\right\}$

Here $\mathfrak{h}_{\epsilon_{j}}=\cup_{y=1}^{t}\left\{\zeta_{y j}\right\}$.

Step 3: For $\kappa_{i}, i=1,2,3, \ldots, l$ HT-SF element related to $\kappa_{i}$ denoted by $\mathfrak{A}_{i}$, is defined as follows:

$\mathfrak{A}_{i}=\bigoplus_{j=1}^{s} w_{j} \mathfrak{h}_{\epsilon_{j}}$.

Step 4: Find score values of $\mathfrak{A}_{i}(i=1,2,3, \ldots, l)$.

Step 5: Order score values of $\mathfrak{A}_{i}(i=1,2,3, \ldots, l)$.

Step 6: Choose the alternative which has maximum score value.

Flowchart of the algorithm is given in Fig. 1.

\section{Illustrative example}

We consider that a university wants for filling the position of one assistant professorship in a department. After the announcement for this vacant position, seven candidates $\kappa_{1}, \kappa_{2}, \ldots, \kappa_{7}$ apply for the position. University rector assigns three experts $\partial_{1}, \partial_{2}$, and $\partial_{3}$ to evaluate alternatives according to criterion $\epsilon_{1}=$ experience, $\epsilon_{2}=$ scientific works, and $\epsilon_{3}=$ quality of the researches. After interview, experts determine the weight vector of the criteria as $(0.35,0.25,0.40)^{T}$.

Step 1: Experts evaluate the alternatives by HT-SFNs corresponding to linguistic variables given in Table 3 for each criteria and $n=4$.

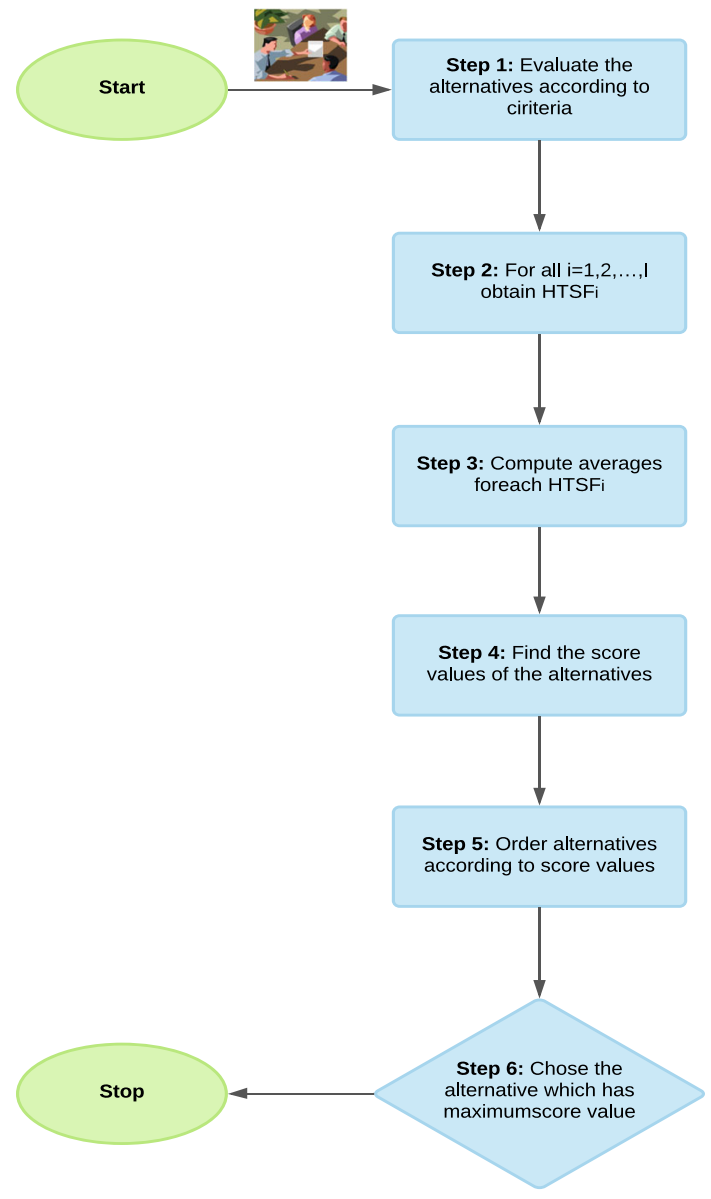

Fig. 1 Flowchart of the proposed method

Table 3 Linguistic variable table for evaluation of the candidates

\begin{tabular}{ll}
\hline Grades & HT-SFNs \\
\hline Very poor (VP) & $(0.100,0.700,0.900)$ \\
Poor (P) & $(0.233,0.634,0.767)$ \\
Medium poor (MP) & $(0.367,0.567,0.634)$ \\
Fairly (F) & $(0.500,0.500,0.500)$ \\
Medium good (MG) & $(0.633,0.436,0.367)$ \\
Good (G) & $(0.764,0.370,0.234)$ \\
Very good (VG) & $(0.900,0.300,0.100)$ \\
\hline
\end{tabular}




\begin{tabular}{|c|c|c|c|c|}
\hline & & $\epsilon_{1}$ & $\epsilon_{2}$ & $\epsilon_{3}$ \\
\hline \multirow[t]{3}{*}{$D_{\kappa_{1}}$} & $\partial_{1}$ & $(0.633,0.436,0.367)$ & $(0.233,0.634,0.767)$ & $*$ \\
\hline & $\partial_{2}$ & $(0.100,0.700,0.900)$ & $(0.900,0.300,0.100)$ & $(0.500,0.500,0.500)$ \\
\hline & $\partial_{3}$ & $*$ & $(0.764,0.370,0.234)$ & $*$ \\
\hline \multirow[t]{3}{*}{$D_{\kappa_{2}}$} & $\partial_{1}$ & * & $(0.233,0.634,0.767)$ & $*$ \\
\hline & $\partial_{2}$ & $(0.233,0.634,0.767)$ & $(0.367,0.567,0.634)$ & $(0.500,0.500,0.500)$ \\
\hline & $\partial_{3}$ & $(0.764,0.370,0.234)$ & $(0.100,0.700,0.900)$ & $(0.633,0.436,0.367)$ \\
\hline \multirow[t]{3}{*}{$D_{\kappa_{3}}$} & $\partial_{1}$ & $(0.367,0.567,0.634)$ & $(0.633,0.436,0.367)$ & $(0.100,0.700,0.900)$ \\
\hline & $\partial_{2}$ & $(0.900,0.300,0.100)$ & $(0.764,0.370,0.234)$ & * \\
\hline & $\partial_{3}$ & $(0.633,0.436,0.367)$ & $(0.100,0.700,0.900)$ & $(0.500,0.500,0.500)$ \\
\hline \multirow[t]{3}{*}{$D_{\kappa_{4}}$} & $\partial_{1}$ & $(0.900,0.300,0.100)$ & $(0.500,0.500,0.500)$ & $(0.100,0.700,0.900)$ \\
\hline & $\partial_{2}$ & $(0.900,0.300,0.100)$ & $*$ & $(0.500,0.500,0.500)$ \\
\hline & $\partial_{3}$ & $*$ & $(0.100,0.700,0.900)$ & $(0.500,0.500,0.500)$ \\
\hline \multirow[t]{3}{*}{$D_{\kappa_{5}}$} & $\partial_{1}$ & $(0.367,0.567,0.634)$ & $(0.500,0.500,0.500)$ & $(0.100,0.700,0.900)$ \\
\hline & $\partial_{2}$ & $(0.100,0.700,0.900)$ & $(0.367,0.567,0.634)$ & $(0.633,0.436,0.367)$ \\
\hline & $\partial_{3}$ & $(0.900,0.300,0.100)$ & $(0.100,0.700,0.900)$ & $(0.500,0.500,0.500)$ \\
\hline \multirow[t]{3}{*}{$D_{\kappa 6}$} & $\partial_{1}$ & $(0.500,0.500,0.500)$ & $(0.633,0.436,0.367)$ & $(0.764,0.370,0.234)$ \\
\hline & $\partial_{2}$ & $*$ & $(0.367,0.567,0.634)$ & $(0.633,0.436,0.367)$ \\
\hline & $\partial_{3}$ & $*$ & $(0.233,0.634,0.767)$ & $(0.500,0.500,0.500)$ \\
\hline \multirow[t]{3}{*}{$D_{\kappa 7}$} & $\partial_{1}$ & $(0.500,0.500,0.500)$ & $(0.633,0.436,0.367)$ & $(0.500,0.500,0.500)$ \\
\hline & $\partial_{2}$ & $(0.367,0.567,0.634)$ & $(0.100,0.700,0.900)$ & $(0.233,0.634,0.767)$ \\
\hline & $\partial_{3}$ & $(0.233,0.634,0.767)$ & $(0.100,0.700,0.900)$ & $*$ \\
\hline
\end{tabular}

Step 2: Using HT-SF decision matrices given in Step 1, $\operatorname{HTSF}_{i}(i=1,2, \ldots, 7)$ are obtained as follows:

$$
\begin{aligned}
& H T S F_{1}=\left\{\left(\epsilon_{1},\{(0.633,0.436,0.367),\right.\right. \\
& \left(\epsilon_{2},\{(0.233,0.634,0.767),\right. \\
& (0.900,0.300,0.100),(0.764,0.370,0.234)\}), \\
& \left.\left(\epsilon_{3},\{(0.500,0.500,0.500)\}\right)\right\}, \\
& H T S F_{2}=\left\{\left(\epsilon_{1},\{(0.764,0.370,0.234),\right.\right. \\
& (0.233,0.634,0.767\}),\left(\epsilon_{2},\{(0.100,0.700,0.900),\right. \\
& (0.233,0.634,0.767),(0.367,0.567,0.634)\}), \\
& \left.\left(\epsilon_{3},\{(0.500,0.500,0.500),(0.633,0.436,0.367)\}\right)\right\}, \\
& H T S F_{3}=\left\{\left(\epsilon_{1},\{(0.367,0.567,0.634),\right.\right. \\
& (0.900,0.300,0.100),(0.633,0.436,0.367)\}), \\
& \left(\epsilon_{2},\{(0.633,0.436,0.367),\right. \\
& (0.764,0.370,0.234),(0.100,0.700,0.900)\}), \\
& \left.\left(\epsilon_{3},\{(0.100,0.700,0.900),(0.500,0.500,0.500)\}\right)\right\}, \\
& H T S F 4=\left\{\left(\epsilon_{1},\{(0.900,0.300,0.100),\right.\right. \\
& (0.900,0.300,0.100)\}),\left(\epsilon_{2},\{(0.100,0.700,0.900),\right. \\
& (0.500,0.500,0.500)\}),\left(\epsilon_{3},\{(0.100,0.700,0.900),\right. \\
& (0.500,0.500,0.500),(0.500,0.500,0.500)\})\},
\end{aligned}
$$




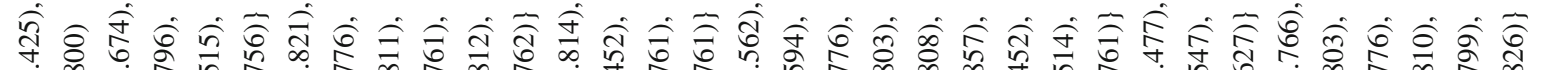

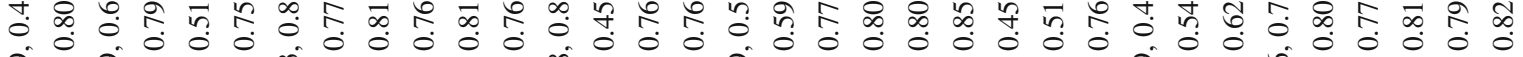

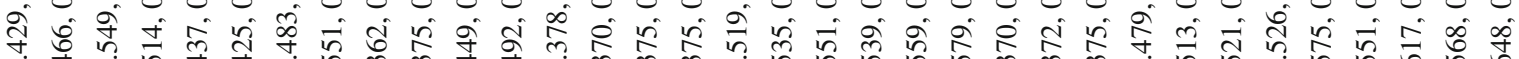

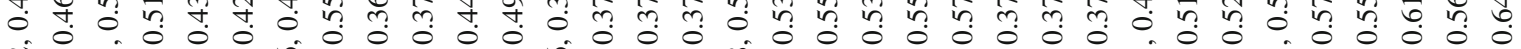

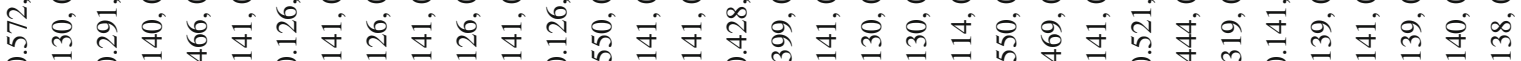

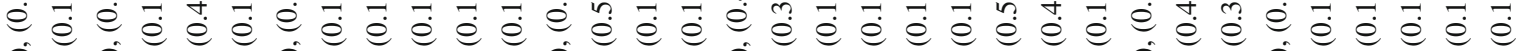

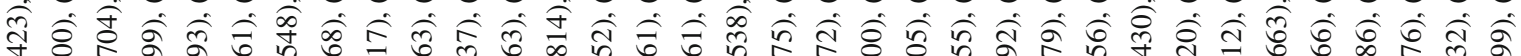

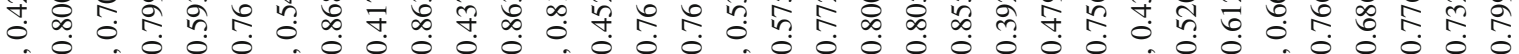

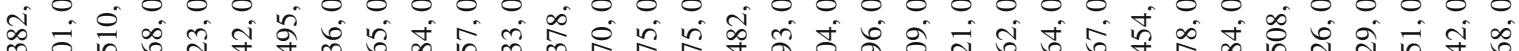
0 t 0 n

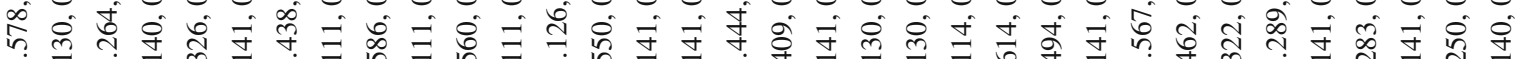

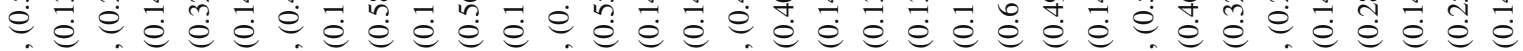

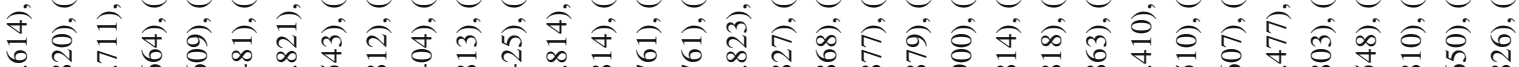

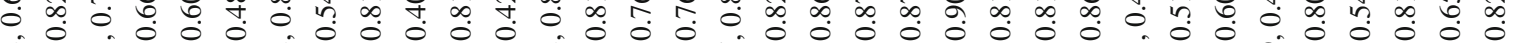

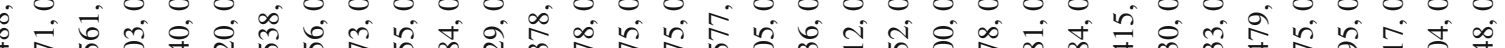

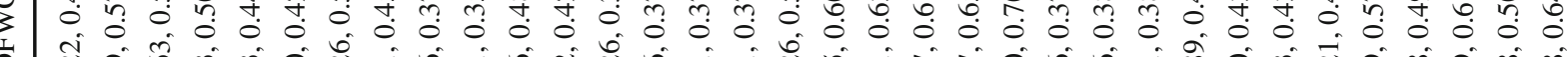

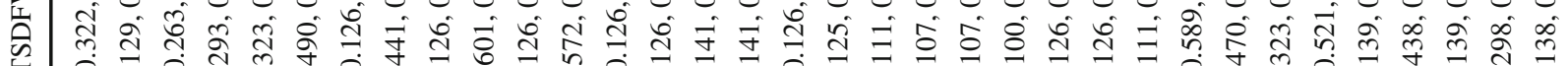

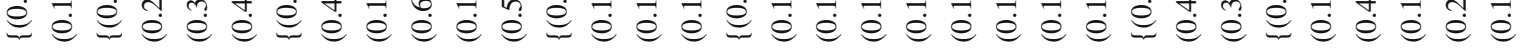

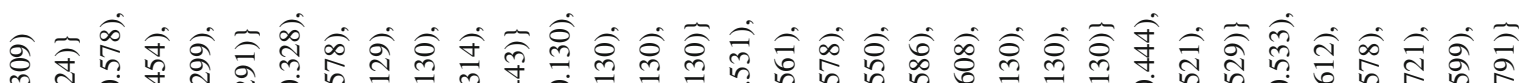

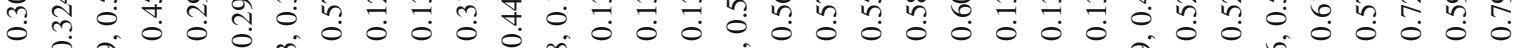

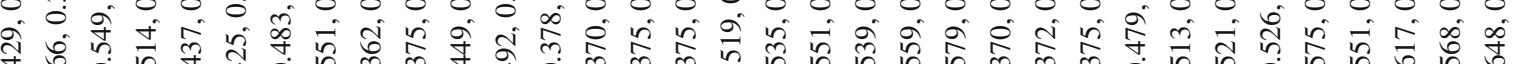

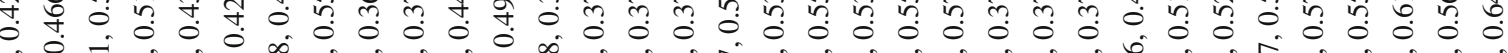

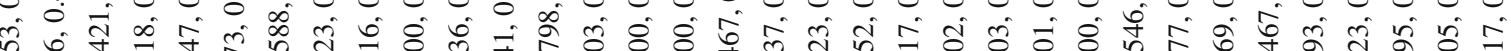
万人

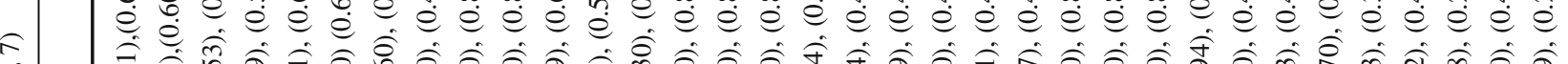

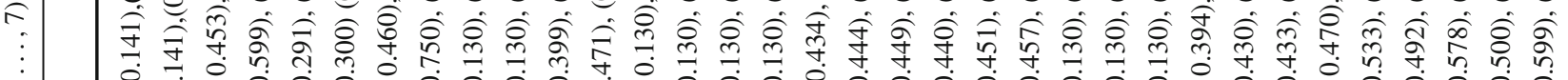

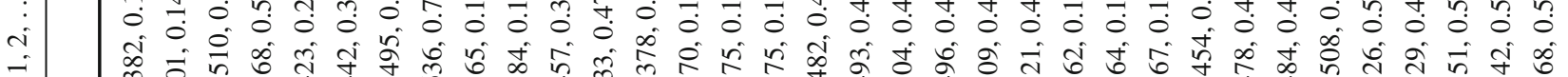

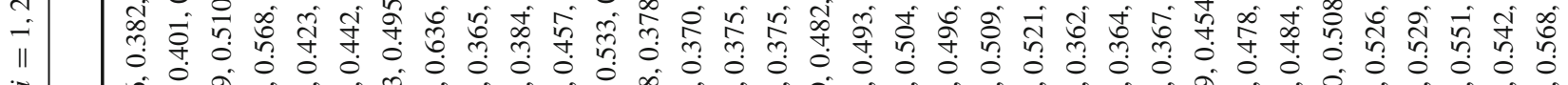

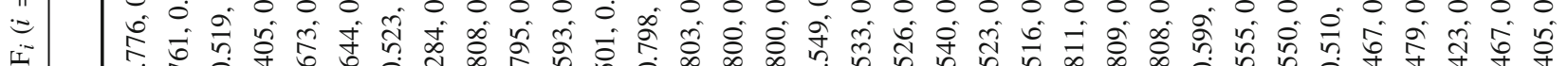

tris

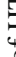
e

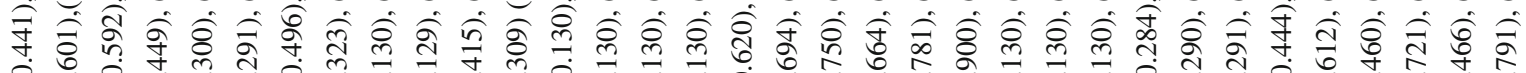

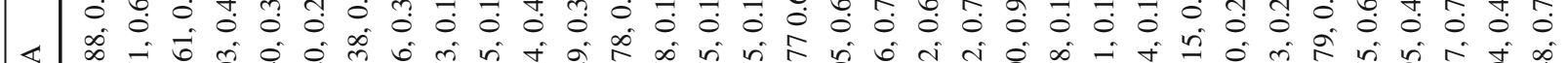

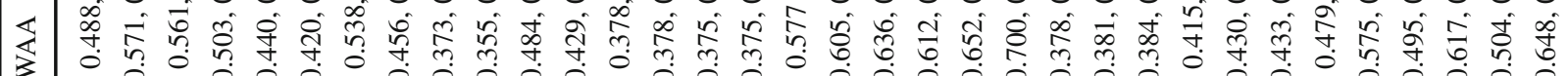

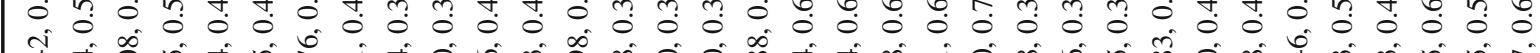
守

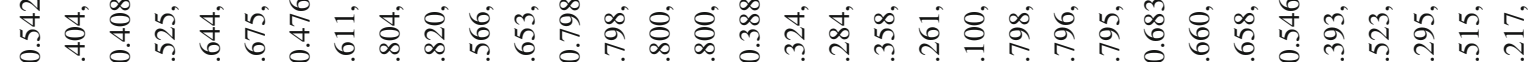
要 สี

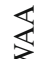

空

空

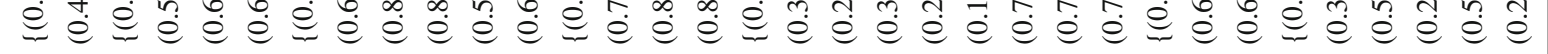

$\frac{\sqrt{0}}{\frac{0}{\circ}}$

$\vec{a} \quad \vec{a}$

बै

$\stackrel{\text { a }}{a}$

ส้

से 
Table 5 Score values of $\mathfrak{A}_{i}$ according to HTSDFWAA and HTSDFWGA values

\begin{tabular}{lcc}
\hline & HTSDFWAA & HTSDFWGA \\
\hline $\mathrm{SV}\left(\mathfrak{A}_{1}\right)$ & 0.156 & -0.208 \\
$\mathrm{SV}\left(\mathfrak{A}_{2}\right)$ & 0.076 & -0.217 \\
$\mathrm{SV}\left(\mathfrak{A}_{3}\right)$ & 0.165 & -0.285 \\
$\mathrm{SV}\left(\mathfrak{A}_{4}\right)$ & 0.409 & -0.305 \\
$\mathrm{SV}\left(\mathfrak{A}_{5}\right)$ & 0.063 & -0.335 \\
$\mathrm{SV}\left(\mathfrak{A}_{6}\right)$ & 0.088 & -0.035 \\
$\mathrm{SV}\left(\mathfrak{A}_{7}\right)$ & -0.104 & -0.319 \\
\hline
\end{tabular}

Step 3: For $n=4$ and $\gamma=1$, HTSDFWAA and HTSDFWGA values of $\operatorname{HTSF}_{i},(i=1,2, \ldots, 7)$ are obtained as in Table 4

Step 4: Score values of $\mathfrak{A}_{i},(i=1,2, \ldots, 7)$ under score function are obtained as in Table 5 .

Step 5: Using Eq. (1), ordering of the candidates is obtained as in Table 6 .

Step 6: From the above illustration, although overall ranking values of the alternatives are different through the use of two operators, optimum alternatives are $\kappa_{4}$ and $\kappa_{6}$ for the two operators, respectively.

\section{Analysis of the effect of parameter $\gamma$ on the results}

To show the effect of the $\gamma$ variable in the formula of HTSDFWAA and HTSDFWGA on MCGDM results, we assign different values to $\gamma$ from 1 to 10 and order candidates according to score values based on HTSDFWAA and HTSDFWGA. Ranking orders of the candidates according to score values and their ranking orders based on HTSDFWAA and HTSDFWGA operators are shown in Table 7. It is clear when $\gamma$ value is changed in the formula HTFD WAS, the optimum candidate is always the same person, and orderings of candidates $\left(\mathrm{SV}\left(\mathfrak{A}_{4}\right)>\mathrm{SV}\left(\mathfrak{A}_{1}\right)>\mathrm{SV}\left(\mathfrak{A}_{3}\right)>\mathrm{SV}\left(\mathfrak{A}_{5}\right)>\right.$ $\left.\mathrm{SV}\left(\mathfrak{A}_{2}\right)>\mathrm{SV}\left(\mathfrak{A}_{6}\right)>\mathrm{SV}\left(\mathfrak{A}_{7}\right)\right)$ are same except in condition $\gamma=1$. By Table 8, it is clear that when the value of $\gamma$ is changed for HTSDFWGA operator, and the ranking orders of candidates $\left(\mathrm{SV}\left(\mathfrak{A}_{6}\right)>\mathrm{SV}\left(\mathfrak{A}_{1}\right)>\mathrm{SV}\left(\mathfrak{A}_{2}\right)>\mathrm{SV}\left(\mathfrak{A}_{3}\right)>\right.$ $\left.\mathrm{SV}\left(\mathfrak{A}_{5}\right)>\mathrm{SV}\left(\mathfrak{A}_{7}\right)>\mathrm{SV}\left(\mathfrak{A}_{4}\right)\right)$ are same except from in case $\gamma=1$. In addition, best suitable candidate is identical for $1 \leq \gamma \leq 10$.

Graphical representation of Table 7 is given in Fig. 2.

Graphical representation of Table 7 is given in Fig. 3.

Using HTSDFWAA operator and score function, we obtain alternative $\mathfrak{A}_{4}$ which has maximum score value as an optimum element. Also, using HTSDFWGA operator and score function of HT-SFEs, we obtain alternative $\mathfrak{A}_{6}$ which has maximum score value. We see that different alternatives which are maximum score values are obtained each of proposed aggregation operators. In Table 5, for $\gamma=2,3,4, \ldots, 10$ alternative $\mathfrak{A}_{4}$ which has minimum score value. In HTSDFWGA operator, since third competent of a TSFE is obtained using Dombi t-conorm and it has a negative effect over score value, alternative $\mathfrak{A}_{4}$ which has minimum score can be considered optimum element. Furthermore, we can give this relation by $1-S \mathfrak{A}_{i}$ for score value obtained using result of HTSDFWGA operator.
Table 6 Score values of $\mathfrak{A}_{i}$ obtained using HTSDFWAA and HTSDFWGA operators

\begin{tabular}{ll}
\hline & Ordering \\
\hline HTSDFWAA & $\mathrm{SV}\left(\mathfrak{A}_{4}\right)>\mathrm{SV}\left(\mathfrak{A}_{3}\right)>\mathrm{SV}\left(\mathfrak{A}_{1}\right)>\mathrm{SV}\left(\mathfrak{A}_{6}\right)>\mathrm{SV}\left(\mathfrak{A}_{2}\right)>\mathrm{SV}\left(\mathfrak{A}_{5}\right)>\mathrm{SV}\left(\mathfrak{A}_{7}\right)$ \\
HTSDFWGA & $\mathrm{SV}\left(\mathfrak{A}_{6}\right)>\mathrm{SV}\left(\mathfrak{A}_{1}\right)>\mathrm{SV}\left(\mathfrak{A}_{2}\right)>\mathrm{SV}\left(\mathfrak{A}_{3}\right)>\mathrm{SV}\left(\mathfrak{A}_{4}\right)>\mathrm{SV}\left(\mathfrak{A}_{7}\right)>\mathrm{SV}\left(\mathfrak{A}_{5}\right)$ \\
\hline
\end{tabular}

Table 7 Ranking order for different $\gamma$ values in the HTSDFWAA operator

\begin{tabular}{lllllllll}
\hline$\gamma$ & $\mathrm{SV}\left(\mathfrak{A}_{1}\right)$ & $\mathrm{SV}\left(\mathfrak{A}_{2}\right)$ & $\mathrm{SV}\left(\mathfrak{A}_{3}\right)$ & $\mathrm{SV}\left(\mathfrak{A}_{4}\right)$ & $\mathrm{SV}\left(\mathfrak{A}_{5}\right)$ & $\mathrm{SV}\left(\mathfrak{A}_{6}\right)$ & $\mathrm{SV}\left(\mathfrak{A}_{7}\right)$ & Ranking order \\
\hline 1 & 0.273 & 0.148 & 0.265 & 0.573 & 0.156 & 0.134 & -0.049 & $\mathrm{SV}\left(\mathfrak{A}_{4}\right)>\mathrm{SV}\left(\mathfrak{A}_{3}\right)>\mathrm{SV}\left(\mathfrak{A}_{1}\right)>\mathrm{SV}\left(\mathfrak{A}_{6}\right)>\mathrm{SV}\left(\mathfrak{A}_{2}\right)>\mathrm{SV}\left(\mathfrak{A}_{5}\right)>\mathrm{SV}\left(\mathfrak{A}_{7}\right)$ \\
2 & 0.237 & 0.125 & 0.235 & 0.530 & 0.130 & 0.118 & -0.067 & $\mathrm{SV} 4\left(\mathfrak{A}_{4}\right)>\mathrm{SV}\left(\mathfrak{A}_{1}\right)>\mathrm{SV}\left(\mathfrak{A}_{3}\right)>\mathrm{SV}\left(\mathfrak{A}_{5}\right)>\mathrm{SV}\left(\mathfrak{A}_{2}\right)>\mathrm{SV}\left(\mathfrak{A}_{6}\right)>\mathrm{SV}\left(\mathfrak{A}_{7}\right)$ \\
3 & 0.273 & 0.148 & 0.265 & 0.573 & 0.156 & 0.134 & -0.049 & $\mathrm{SV}\left(\mathfrak{A}_{4}\right)>\mathrm{SV}\left(\mathfrak{A}_{1}\right)>\mathrm{SV}\left(\mathfrak{A}_{3}\right)>\mathrm{SV}\left(\mathfrak{A}_{5}\right)>\mathrm{SV}\left(\mathfrak{A}_{2}\right)>\mathrm{SV}\left(\mathfrak{A}_{6}\right)>\mathrm{SV}\left(\mathfrak{A}_{7}\right)$ \\
4 & 0.292 & 0.161 & 0.281 & 0.595 & 0.170 & 0.143 & -0.040 & $\mathrm{SV}\left(\mathfrak{A}_{4}\right)>\mathrm{SV}\left(\mathfrak{A}_{1}\right)>\mathrm{SV}\left(\mathfrak{A}_{3}\right)>\mathrm{SV}\left(\mathfrak{A}_{5}\right)>\mathrm{SV}\left(\mathfrak{A}_{2}\right)>\mathrm{SV}\left(\mathfrak{A}_{6}\right)>\mathrm{SV}\left(\mathfrak{A}_{7}\right)$ \\
5 & 0.305 & 0.169 & 0.291 & 0.607 & 0.178 & 0.149 & -0.033 & $\mathrm{SV}\left(\mathfrak{A}_{4}\right)>\mathrm{SV}\left(\mathfrak{A}_{1}\right)>\mathrm{SV}\left(\mathfrak{A}_{3}\right)>\mathrm{SV}\left(\mathfrak{A}_{5}\right)>\mathrm{SV}\left(\mathfrak{A}_{2}\right)>\mathrm{SV}\left(\mathfrak{A}_{6}\right)>\mathrm{SV}\left(\mathfrak{A}_{7}\right)$ \\
6 & 0.313 & 0.175 & 0.298 & 0.615 & 0.183 & 0.153 & -0.029 & $\mathrm{SV}\left(\mathfrak{A}_{4}\right)>\mathrm{SV}\left(\mathfrak{A}_{1}\right)>\mathrm{SV}\left(\mathfrak{A}_{3}\right)>\mathrm{SV}\left(\mathfrak{A}_{5}\right)>\mathrm{SV}\left(\mathfrak{A}_{2}\right)>\mathrm{SV}\left(\mathfrak{A}_{6}\right)>\mathrm{SV}\left(\mathfrak{A}_{7}\right)$ \\
7 & 0.319 & 0.179 & 0.303 & 0.621 & 0.187 & 0.157 & -0.026 & $\mathrm{SV}\left(\mathfrak{A}_{4}\right)>\mathrm{SV}\left(\mathfrak{A}_{1}\right)>\mathrm{SV}\left(\mathfrak{A}_{3}\right)>\mathrm{SV}\left(\mathfrak{A}_{5}\right)>\mathrm{SV}\left(\mathfrak{A}_{2}\right)>\mathrm{SV}\left(\mathfrak{A}_{6}\right)>\mathrm{SV}\left(\mathfrak{A}_{7}\right)$ \\
8 & 0.323 & 0.182 & 0.307 & 0.626 & 0.190 & 0.159 & -0.023 & $\mathrm{SV}\left(\mathfrak{A}_{4}\right)>\mathrm{SV}\left(\mathfrak{A}_{1}\right)>\mathrm{SV}\left(\mathfrak{A}_{3}\right)>\mathrm{SV}\left(\mathfrak{A}_{5}\right)>\mathrm{SV}\left(\mathfrak{A}_{2}\right)>\mathrm{SV}\left(\mathfrak{A}_{6}\right)>\mathrm{SV}\left(\mathfrak{A}_{7}\right)$ \\
9 & 0.327 & 0.185 & 0.310 & 0.629 & 0.192 & 0.161 & -0.022 & $\mathrm{SV}\left(\mathfrak{A}_{4}\right)>\mathrm{SV}\left(\mathfrak{A}_{1}\right)>\mathrm{SV}\left(\mathfrak{A}_{3}\right)>\mathrm{SV}\left(\mathfrak{A}_{5}\right)>\mathrm{SV}\left(\mathfrak{A}_{2}\right)>\mathrm{SV}\left(\mathfrak{A}_{6}\right)>\mathrm{SV}\left(\mathfrak{A}_{7}\right)$ \\
10 & 0.330 & 0.187 & 0.312 & 0.632 & 0.194 & 0.162 & -0.020 & $\mathrm{SV}\left(\mathfrak{A}_{4}\right)>\mathrm{SV}\left(\mathfrak{A}_{1}\right)>\mathrm{SV}\left(\mathfrak{A}_{3}\right)>\mathrm{SV}\left(\mathfrak{A}_{5}\right)>\mathrm{SV}\left(\mathfrak{A}_{2}\right)>\mathrm{SV}\left(\mathfrak{A}_{6}\right)>\mathrm{SV}\left(\mathfrak{A}_{7}\right)$ \\
\hline
\end{tabular}


Table 8 Ranking order for different $\gamma$ values in the HTSDFWGA operator

\begin{tabular}{lllllllll}
\hline$\gamma$ & $\mathrm{SV}\left(\mathfrak{A}_{1}\right)$ & $\mathrm{SV}\left(\mathfrak{A}_{2}\right)$ & $\mathrm{SV}\left(\mathfrak{A}_{3}\right)$ & $\mathrm{SV}\left(\mathfrak{A}_{4}\right)$ & $\mathrm{SV}\left(\mathfrak{A}_{5}\right)$ & $\mathrm{SV}\left(\mathfrak{A}_{6}\right)$ & $\mathrm{SV}\left(\mathfrak{A}_{7}\right)$ & $\mathrm{Ranking}$ order \\
\hline 1 & -0.316 & -0.333 & -0.392 & -0.465 & -0.435 & -0.099 & -0.434 & $\mathrm{SV}\left(\mathfrak{A}_{6}\right)>\mathrm{SV}\left(\mathfrak{A}_{1}\right)>\mathrm{SV}\left(\mathfrak{A}_{2}\right)>\mathrm{SV}\left(\mathfrak{A}_{3}\right)>\mathrm{SV}\left(\mathfrak{A}_{4}\right)>\mathrm{SV}\left(\mathfrak{A}_{7}\right)>\mathrm{SV}\left(\mathfrak{A}_{5}\right)$ \\
2 & -0.284 & -0.297 & -0.363 & -0.422 & -0.406 & -0.076 & -0.395 & $\mathrm{SV}\left(\mathfrak{A}_{6}\right)>\mathrm{SV}\left(\mathfrak{A}_{1}\right)>\mathrm{SV}\left(\mathfrak{A}_{2}\right)>\mathrm{SV}\left(\mathfrak{A}_{3}\right)>\mathrm{SV}\left(\mathfrak{A}_{7}\right)>\mathrm{SV}\left(\mathfrak{A}_{5}\right)>\mathrm{SV}\left(\mathfrak{A}_{4}\right)$ \\
3 & -0.316 & -0.333 & -0.392 & -0.465 & -0.435 & -0.099 & -0.434 & $\mathrm{SV}\left(\mathfrak{A}_{6}\right)>\mathrm{SV}\left(\mathfrak{A}_{1}\right)>\mathrm{SV}\left(\mathfrak{A}_{2}\right)>\mathrm{SV}\left(\mathfrak{A}_{3}\right)>\mathrm{SV}\left(\mathfrak{A}_{7}\right)>\mathrm{SV}\left(\mathfrak{A}_{5}\right)>\mathrm{SV}\left(\mathfrak{A}_{4}\right)$ \\
4 & -0.333 & -0.352 & -0.408 & -0.486 & -0.449 & -0.113 & -0.455 & $\mathrm{SV}\left(\mathfrak{A}_{6}\right)>\mathrm{SV}\left(\mathfrak{A}_{1}\right)>\mathrm{SV}\left(\mathfrak{A}_{2}\right)>\mathrm{SV}\left(\mathfrak{A}_{3}\right)>\mathrm{SV}\left(\mathfrak{A}_{5}\right)>\mathrm{SV}\left(\mathfrak{A}_{7}\right)>\mathrm{SV}\left(\mathfrak{A}_{4}\right)$ \\
5 & -0.344 & -0.364 & -0.417 & -0.498 & -0.458 & -0.123 & -0.468 & $\mathrm{SV}\left(\mathfrak{A}_{6}\right)>\mathrm{SV}\left(\mathfrak{A}_{1}\right)>\mathrm{SV}\left(\mathfrak{A}_{2}\right)>\mathrm{SV}\left(\mathfrak{A}_{3}\right)>\mathrm{SV}\left(\mathfrak{A}_{5}\right)>\mathrm{SV}\left(\mathfrak{A}_{7}\right)>\mathrm{SV}\left(\mathfrak{A}_{4}\right)$ \\
6 & -0.350 & -0.372 & -0.423 & -0.507 & -0.464 & -0.129 & -0.477 & $\mathrm{SV}\left(\mathfrak{A}_{6}\right)>\mathrm{SV}\left(\mathfrak{A}_{1}\right)>\mathrm{SV}\left(\mathfrak{A}_{2}\right)>\mathrm{SV}\left(\mathfrak{A}_{3}\right)>\mathrm{SV}\left(\mathfrak{A}_{5}\right)>\mathrm{SV}\left(\mathfrak{A}_{7}\right)>\mathrm{SV}\left(\mathfrak{A}_{4}\right)$ \\
7 & -0.355 & -0.378 & -0.427 & -0.512 & -0.468 & -0.133 & -0.484 & $\mathrm{SV}\left(\mathfrak{A}_{6}\right)>\mathrm{SV}\left(\mathfrak{A}_{1}\right)>\mathrm{SV}\left(\mathfrak{A}_{2}\right)>\mathrm{SV}\left(\mathfrak{A}_{3}\right)>\mathrm{SV}\left(\mathfrak{A}_{5}\right)>\mathrm{SV}\left(\mathfrak{A}_{7}\right)>\mathrm{SV}\left(\mathfrak{A}_{4}\right)$ \\
8 & -0.359 & -0.383 & -0.431 & -0.517 & -0.472 & -0.137 & -0.489 & $\mathrm{SV}\left(\mathfrak{A}_{6}\right)>\mathrm{SV}\left(\mathfrak{A}_{1}\right)>\mathrm{SV}\left(\mathfrak{A}_{2}\right)>\mathrm{SV}\left(\mathfrak{A}_{3}\right)>\mathrm{SV}\left(\mathfrak{A}_{5}\right)>\mathrm{SV}\left(\mathfrak{A}_{7}\right)>\mathrm{SV}\left(\mathfrak{A}_{4}\right)$ \\
9 & -0.362 & -0.386 & -0.433 & -0.520 & -0.474 & -0.140 & -0.492 & $\mathrm{SV}\left(\mathfrak{A}_{6}\right)>\mathrm{SV}\left(\mathfrak{A}_{1}\right)>\mathrm{SV}\left(\mathfrak{A}_{2}\right)>\mathrm{SV}\left(\mathfrak{A}_{3}\right)>\mathrm{SV}\left(\mathfrak{A}_{5}\right)>\mathrm{SV}\left(\mathfrak{A}_{7}\right)>\mathrm{SV}\left(\mathfrak{A}_{4}\right)$ \\
10 & -0.364 & -0.389 & -0.435 & -0.523 & -0.476 & -0.142 & -0.495 & $\mathrm{SV}\left(\mathfrak{A}_{6}\right)>\mathrm{SV}\left(\mathfrak{A}_{1}\right)>\mathrm{SV}\left(\mathfrak{A}_{2}\right)>\mathrm{SV}\left(\mathfrak{A}_{3}\right)>\mathrm{SV}\left(\mathfrak{A}_{5}\right)>\mathrm{SV}\left(\mathfrak{A}_{7}\right)>\mathrm{SV}\left(\mathfrak{A}_{4}\right)$
\end{tabular}

Fig. 2 Ranking order for different $\gamma$ values in the HTSDFWAA operator

Fig. 3 Ranking order for different $\gamma$ values in the HTSDFWGA operator

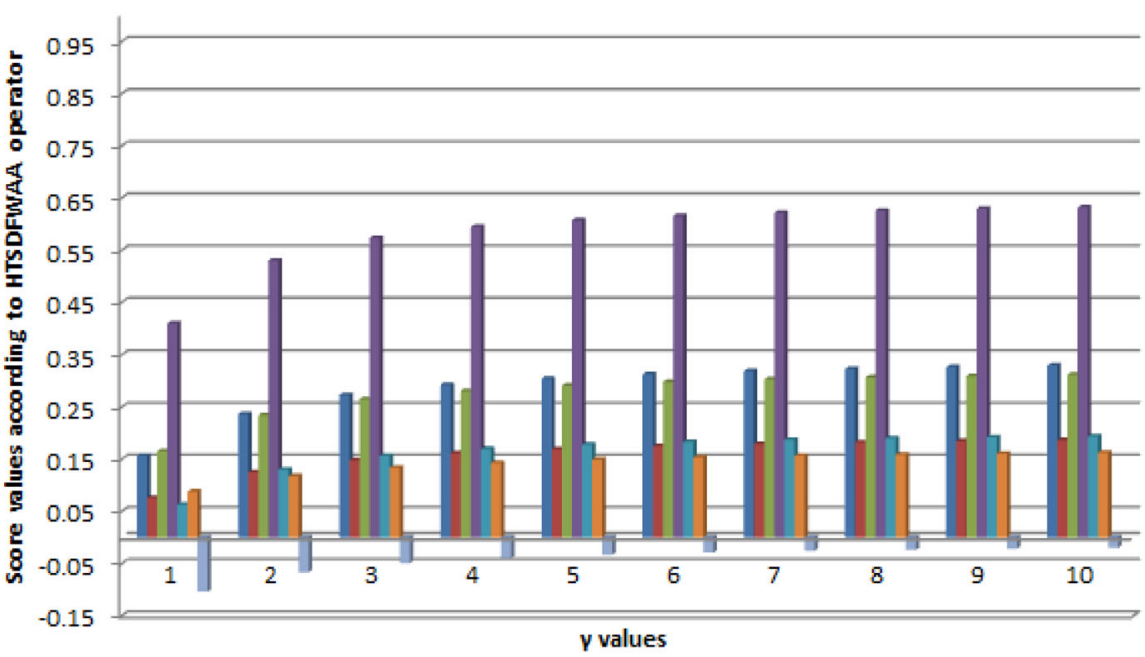

a1

$\square \mathrm{A} 2$

$\mathrm{A} 3$

口4

A4

A6

A7

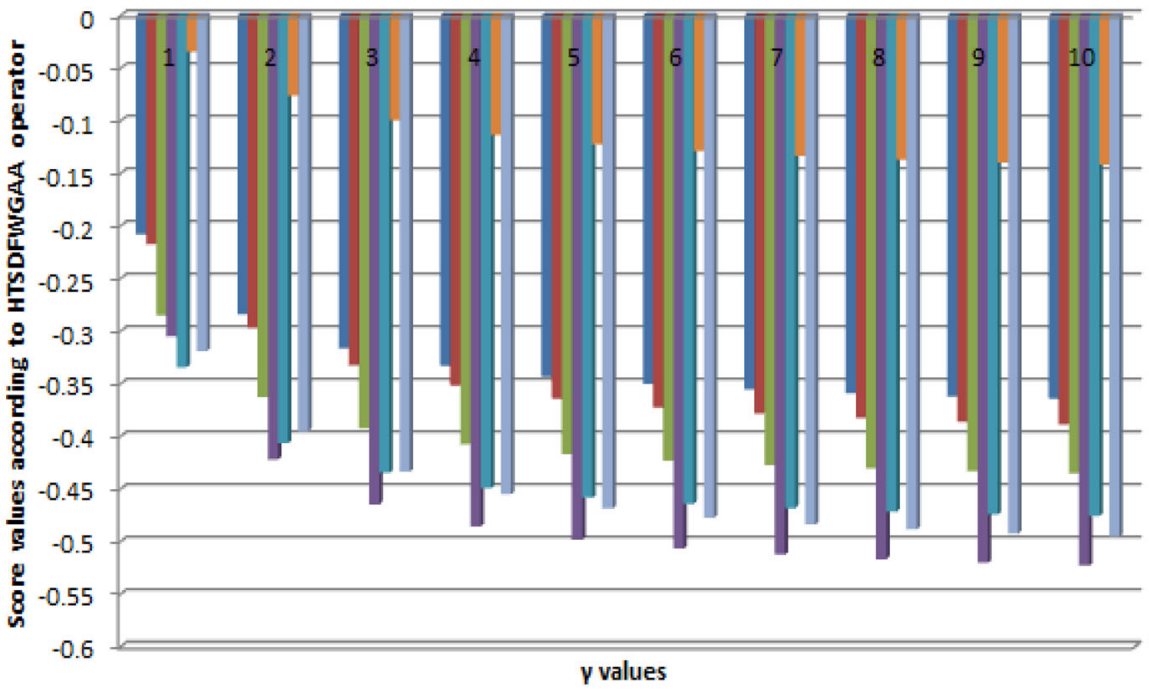

ㄱ1 
Table 9 Comparison table of the HT-SFS with some extensions of fuzzy

\begin{tabular}{|c|c|c|c|c|c|c|}
\hline & \multicolumn{3}{|c|}{$n$ (degrees of component) } & \multicolumn{2}{|c|}{$k$ (length) } & \multirow[t]{2}{*}{ Condition } \\
\hline & 1 & 2 & $>2$ & 1 & $>1$ & \\
\hline Fuzzy set [1] & $\checkmark$ & $x$ & $x$ & $\checkmark$ & $x$ & $0 \leq s_{k}+d_{k}=1, i_{k}=0$ \\
\hline Intuitionistic fuzzy set [4] & $\checkmark$ & $x$ & $x$ & $\checkmark$ & $x$ & $s_{k}+i_{k}+d_{k}=1$ \\
\hline Pythagorean fuzzy set [5] & $\checkmark$ & $\checkmark$ & $x$ & $\checkmark$ & $x$ & $0 \leq s_{k}^{2}+d_{k}^{2} \leq 1$ \\
\hline Picture fuzzy set [7] & $\checkmark$ & $x$ & $x$ & $\checkmark$ & $x$ & $0 \leq s_{k}+i_{k}+d_{k} \leq 1$ \\
\hline Spherical fuzzy set [25] & $\checkmark$ & $\checkmark$ & $x$ & $\checkmark$ & $x$ & $0 \leq s_{k}^{2}+i_{k}^{2}+d_{k}^{2} \leq 1$ \\
\hline q-rung orthopair fuzzy set [6] & $\checkmark$ & $\checkmark$ & $\checkmark$ & $\checkmark$ & $x$ & $0 \leq s_{k}^{n}+d_{k}^{n} \leq 1$ \\
\hline T-spherical fuzzy set [27] & $\checkmark$ & $\checkmark$ & $\checkmark$ & $\checkmark$ & $x$ & $0 \leq s_{k}^{n}+i_{k}^{n}+d_{k}^{n} \leq 1$ \\
\hline Hesitant fuzzy set $[42,43]$ & $\checkmark$ & $\times$ & $x$ & $\checkmark$ & $\checkmark$ & $0 \leq s_{k}+d_{k}=1, i_{k}=0$ \\
\hline Intuitionistic hesitant fuzzy set [75] & $\checkmark$ & $x$ & $x$ & $\checkmark$ & $\checkmark$ & $0 \leq s_{k}+d_{k}=1$ \\
\hline Hesitant Pythagorean fuzzy set [76] & $\checkmark$ & $\checkmark$ & $\times$ & $\checkmark$ & $\checkmark$ & $0 \leq s_{k}^{2}+d_{k}^{2} \leq 1$ \\
\hline q-rung Orthopair hesitant fuzzy set [77] & $\checkmark$ & $\checkmark$ & $\checkmark$ & $\checkmark$ & $\checkmark$ & $0 \leq s_{k}^{n}+d_{k}^{n} \leq 1$ \\
\hline Picture hesitant fuzzy set [59] & $\checkmark$ & $\checkmark$ & $x$ & $\checkmark$ & $\checkmark$ & $0 \leq s_{k}+i_{k}+d_{k} \leq 1$ \\
\hline Hesitant T-spherical fuzzy set & $\checkmark$ & $\checkmark$ & $\checkmark$ & $\checkmark$ & $\checkmark$ & $0 \leq s_{k}^{n}+i_{k}^{n}+d_{k}^{n} \leq 1$ \\
\hline
\end{tabular}

\section{Comparative analysis and discussion}

In this section, we compare HT-SFS with other extensions of the fuzzy sets. Let $\mathbb{T}_{H}=\left\{\left(x,\left\{\left(s_{k}, i_{k}, d_{k}\right): 1 \leq k \leq l_{x}\right\}\right)\right.$ : $x \in \mathfrak{X}\}$. Then, comparison table can be given as in Table 9.

Here, we see that HT-SFS is an extension of sets specified in the Table 9. Therefore, the set structure defined in this paper has advantages of the other extensions of fuzzy sets specified in Table 9 in the modelling. It also models some problems which cannot be modelled existing set theories. In the following example, relation between these sets is explained.

\section{Conclusion}

In this paper, the concept of hesitant T-spherical sets and its set theoretical operations such as union, intersection, and complement have been defined. To be more understandable, some examples are given related to defined operations. Based on Dombi t-norm and Domb t-conorm operations, arithmetic operations between two HT-SFEs and

some aggregation operators such as HTSDFWAA, HTSDFWGA, HTSDFOWAA, and HTSDFOWGA operators have been introduced. Furthermore, an MCGDM method has been developed and presented an application to MCGDM problem involving selecting a person for a vacant position in any department of the university. Obtained results have been compared for different parameters. Also, the proposed set structure has been compared by other extensions of the fuzzy sets and mentioned its advantages. In future, our targets are to study on other aggregation operators, similarity measures, distance measures, and decision-making method based on TOPSIS, VIKOR, AHP, etc.

Author Contributions This article was produced from M.Sc thesis of AHSA-H [78]. AHSA-H developed the theoretical formalism, and gave the examples. Both AHSA-H and FK contributed to the final version of the manuscript. FK supervised the manuscript.

Funding This research received no specific grant from any funding agency in the public, commercial, or not-for-profit sectors.

\section{Declarations}

Conflict of interest The authors declare no conflict of interest.

Open Access This article is licensed under a Creative Commons Attribution 4.0 International License, which permits use, sharing, adaptation, distribution and reproduction in any medium or format, as long as you give appropriate credit to the original author(s) and the source, provide a link to the Creative Commons licence, and indicate if changes were made. The images or other third party material in this article are included in the article's Creative Commons licence, unless indicated otherwise in a credit line to the material. If material is not included in the article's Creative Commons licence and your intended use is not permitted by statutory regulation or exceeds the permitted use, you will need to obtain permission directly from the copyright holder. To view a copy of this licence, visit http://creativecomm ons.org/licenses/by/4.0/.

\section{References}

1. Zadeh LA (1965) Fuzzy sets. Inf Control 8(3):338-353

2. Gadekallu TR, Gao X-Z (2021) An efficient attribute reduction and fuzzy logic classifier for heart disease and diabetes prediction. Recent Adv Comput Sci Commun 14(1):158-165

3. Sakthidasan K, Gao X-Z, Devabalaji KR, Roopa TM (2021) Energy based random repeat trust computation approach and reliable fuzzy 
and heuristic ant colony mechanism for improving QoS in WSN. Energy Rep. https://doi.org/10.1016/egyr.2021.08.121

4. Atanassov KT (1986) Intuitionistic fuzzy sets. Fuzzy Sets Syst 20(1):87-96

5. Yager RR Pythagorean fuzzy subsets (2013) Joint IFSA World Congress and NAFIPS Annual Meeting (IFSA/NAFIPS) 57-61

6. Yager RR (2013) Pythagorean membership grades in multi-criteria decision making. IEEE Trans Fuzzy Syst 22(4):958-965

7. Cuong BC (2013) Picture fuzzy sets-First results, Part 1, In Seminar Neuro-Fuzzy Systems with Applications; Institute of Mathematics. Hanoi, Vietnam, Vietnam Academy of Science and Technology

8. Cuong BC (2013) Picture fuzzy sets-First results, Part 2, In Seminar Neuro-Fuzzy Systems with Applications; Institute of Mathematics. Hanoi, Vietnam, Vietnam Academy of Science and Technology

9. Garg H (2017) Some picture fuzzy aggregation operators and their applications to multicriteria decision-making. Arab J Sci Eng 42(12):5275-5290

10. Peng X, Dai J (2017) Algorithm for picture fuzzy multiple attribute decision-making based on new distance measure. Int J Uncertain Quantif 7(2):177-187

11. Wei G (2017) Picture fuzzy aggregation operators and their application to multiple attribute decision making. J Intell Fuzzy Syst 33(2):713-724

12. Wei G (2018) TODIM method for picture fuzzy multiple attribute decision making. Informatica 29(3):555-566

13. Cao G (2020) A multi-criteria picture fuzzy decision-making model for green supplier selection based on fractional programming. Int J Comput Commun Control 15(1):1-14

14. Joshi R (2020) A novel decision-making method using r-norm concept and VIKOR approach under picture fuzzy environment. Expert Syst Appl 147:113228

15. Tian C, Peng J, Zhang W, Zhang S, Wang J (2020) Tourism environmental impact assessment based on improved AHP and picture fuzzy PROMETHEE II methods. Technol Econ Dev Econ 26(2):355-378

16. Wei G (2017) Some cosine similarity measures for picture fuzzy sets and their applications to strategic decision making. Informatica 28(3):547-564

17. Wei G, Gao H (2018) The generalized Dice similarity measures for picture fuzzy sets and their applications. Informatica 29(1):107124

18. Wei G (2018) Some similarity measures for picture fuzzy sets and their applications. Iran J Fuzzy Syst 15(1):77-89

19. Rafiq M, Ashraf S, Abdullah S, Mahmood T, Muhammad S (2019) The cosine similarity measures of spherical fuzzy sets and their applications in decision making. J Intell Fuzzy Syst 36(6):60596073

20. Thao NX (2020) Similarity measures of picture fuzzy sets based on entropy and their application in MCDM. Pattern Anal Appl 23(3):1203-1213

21. Singh P (2015) Correlation coefficients for picture fuzzy sets. J Intell Fuzzy Syst 28(2):591-604

22. Ganie AH, Singh S, Bhatia PK (2020) Some new correlation coefficients of picture fuzzy sets with applications. Neural Comput Appl 32:12609-12625

23. Son LH (2016) Generalized picture distance measure and applications to picture fuzzy clustering. Appl Soft Comput 46(C):284-295

24. Hao ND, Son LH, Thong PH (2016) Some improvements of fuzzy clustering algorithms using picture fuzzy sets and applications for geographic data clustering. VNU J Sci Comput Sci Commun Eng 32(3):32-38

25. Gündogdu FK, Kahraman C (2019) Spherical fuzzy sets and spherical fuzzy TOPSIS method. J Intell Fuzzy Syst 36(1):337-352

26. Gündogdu FK, Kahraman C (2020) Spherical fuzzy sets and decision making applications. In: Kahraman C, Cebi S, Cevik Onar S, Oztaysi B, Tolga A, Sari I (eds) Intelligent and fuzzy techniques in big data analytics and decision making. INFUS 2019. Advances in intelligent systems and computing. Springer, Cham, p 1029

27. Mahmood T, Ullah K, Khan Q, Jan N (2019) An approach toward decision-making and medical diagnosis problems using the concept of spherical fuzzy sets. Neural Comput. Appl. 31:7041-7053

28. Ullah K, Mahmood T, Jan N (2018) Similarity measures for T-spherical fuzzy sets with applications in pattern recognition. Symmetry 10(6): 193

29. Garg H, Munir M, Ullah K, Mahmood T, Jan N (2018) Algorithm for T-spherical fuzzy multi-attribute decision making based on improved interactive aggregation operators. Symmetry 10(12):670

30. Ullah K, Mahmood T, Jan N, Ali Z (2018) A note on geometric aggregation operators in T-spherical fuzzy environment and their applications in multi-attribute decision making. J Eng Appl Sci 37(2):75-86

31. Ullah K, Hassan N, Mahmood T, Jan N, Hassan M (2019) Evaluation of investment policy based on multi-attribute decision-making using interval valued T-spherical fuzzy aggregation operators. Symmetry 11(3):357

32. Liu P, Khan Q, Mahmood T, Hassan N (2019) T-spherical fuzzy power muirhead mean operator based on novel operational laws and their application in multi-attribute group decision making. IEEE Access 7:22613-22632

33. Wu M, Chen T, Fan J (2020) Divergence measure of T-spherical fuzzy sets and its applications in pattern recognition. IEEE Access 8:10208-10221

34. Zeng S, Garg H, Munir M, Mahmood T, Hussain A (2019) A multiattribute decision making process with immediate probabilistic interactive averaging aggregation operators of T-spherical fuzzy sets and its application in the selection of solar cells. Energies 12(23):4436

35. Guleria A, Bajaj RK (2019) T-spherical fuzzy soft sets and its aggregation operators with application in decision making. Sci Iran. https://doi.org/10.24200/SCI.2019.53027.3018

36. Quek SG, Selvachandran G, Munir M, Mahmood T, Ullah K, Son LH, Thong PH, Kumar R, Priyadarshini I (2019) Multiattribute multi-perception decision-making based on generalized T-spherical fuzzy weighted aggregation operators on neutrosophic sets. Mathematics 7:780

37. Munir M, Kalsoom H, Ullah K, Mahmood T, Chu YM (2020) T-spherical fuzzy Einstein hybrid aggregation operators and their applications in multi-attribute decision making problems. Symmetry $12(3): 365$

38. Ullah K, Garg H, Mahmood T, Jan N, Ali Z (2020) Correlation coefficients for T-spherical fuzzy sets and their applications in clustering and multi-attribute decision making. Soft Comput 24(3):1647-1659

39. Ullah K, Mahmood T, Garg H (2020) Evaluation of the performance of search and rescue robots using T-spherical fuzzy hamacher aggregation operators. Int J Fuzzy Syst 22(2):570-582

40. Ali Z, Mahmood T, Yang MS (2020) Complex T-spherical fuzzy aggregation operators with application to multi-attribute decision making. Symmetry 12(8):1311

41. Ozlu S, Karaaslan F (2021) Correlation coefficient of T-spherical type-2 hesitant fuzzy sets and their applications in clustering analysis. J Ambient Intell Hum Comput. https://doi.org/10.1007/ s12652-021-02904-8

42. Torra V, Narukawa Y On hesitant fuzzy sets and decision. In: 2009 IEEE international conference on fuzzy systems. IEEE, pp 13781382

43. Torra V (2010) Hesitant fuzzy sets. Int J Intell Syst 25(6):529-539

44. Xu Z, Xia M (2011) On distance and correlation measures of hesitant fuzzy information. Int J Intell Syst 26(5):410-425

45. Li D, Zeng W, Li J (2015) New distance and similarity measures on hesitant fuzzy sets and their applications in multiple criteria decision making. Eng Appl Artif Intell 40:11-16 
46. Zeng S, Xiao Y (2018) A method based on TOPSIS and distance measures for hesitant fuzzy multiple attribute decision making. Technol Econ Dev Econ 24(3):969-983

47. Hu J, Yang Y, Zhang X, Chen X (2018) Similarity and entropy measures for hesitant fuzzy sets. Int Trans Oper Res 25(3):857886

48. Xu Z, Xia M (2011) Distance and similarity measures for hesitant fuzzy sets. Inf Sci 181(11):2128-2138

49. Zeng W, Li D, Yin Q (2016) Distance and similarity measures between hesitant fuzzy sets and their application in pattern recognition. Pattern Recognit Lett 84:267-271

50. Xia M, Xu Z, Chen N (2013) Some hesitant fuzzy aggregation operators with their application in group decision making. Group Decis Negot 22:259-279

51. Chen N, Xu Z, Xia M (2013) Interval-valued hesitant preference relations and their applications to group decision making. Knowl Based Syst 37:528-540

52. Peng DH, Wang TD, Gao CY, Wang H (2014) Continuous hesitant fuzzy aggregation operators and their application to decision making under interval-valued hesitant fuzzy setting. Sci World J 2014:897304. https://doi.org/10.1155/2014.897304

53. Mu Z, Zeng S, Baležentis T (2015) A novel aggregation principle for hesitant fuzzy elements. Knowl Based Syst 84:134-143

54. Amin F, Fahmi A, Abdullah S, Ali A, Ahmad R, Ghani F (2018) Triangular cubic linguistic hesitant fuzzy aggregation operators and their application in group decision making. J Intell Fuzzy Syst 34(4):2401-2416

55. Fahmi A, Abdullah S, Amin F, Ali A, Ahmed R, Shakeel M (2019) Trapezoidal cubic hesitant fuzzy aggregation operators and their application in group decision-making. J Intell Fuzzy Syst 36(4):3619-3635

56. Jiang C, Jiang S, Chen J (2019) Interval-valued dual hesitant fuzzy hamacher aggregation operators for multiple attribute decision making. J Syst Sci Inf 7(3):227-256

57. Liu HB, Liu Y, Xu L (2020) Dombi interval-valued hesitant fuzzy aggregation operators for information security risk assessment. Math Probl Eng. https://doi.org/10.1155/2020/3198645

58. Zeng W, Xi Y, Yin Q, Guo P (2018) Weighted dual hesitant fuzzy sets and its application in group decision making. In: 2018 14th international conference on computational intelligence and security (CIS). IEEE, pp 77-82

59. Wang R, Li Y (2018) Picture hesitant fuzzy set and its application to multiple criteria decision-making. Symmetry 10(7):295

60. Liang D, Darko AP, Xu Z, Wang M (2019) Aggregation of dual hesitant fuzzy heterogenous related information with extended Bonferroni mean and its application to MULTIMOORA. Comput Ind Eng 135:156-176

61. Liu Y, Rodriguez RM, Alcantud JCR, Qin K, Martinez L (2019) Hesitant linguistic expression soft sets: application to group decision making. Comput Ind Eng 136:575-590

62. Qiao J (2019) Hesitant relations: novel properties and applications in three-way decisions. Inf Sci 497:165-188

63. Bai W, Ding J, Zhang C (2020) Dual hesitant fuzzy graphs with applications to multi-attribute decision making. Int J Cogn Comput Eng 1:18-26

64. Ding Q, Wang YM, Goh M (2020) An extended TODIM approach for group emergency decision making based on bidirectional projection with hesitant triangular fuzzy sets. Comput Ind Eng $151: 106959$

65. Mo X, Zhao H, Xu Z (2020) Feature-based hesitant fuzzy aggregation method for satisfaction with life scale. Appl Soft Comput 94:106493
66. Liao H, Jiang L, Fang R, Qin R (2020) A consensus measure for group decision making with hesitant linguistic preference information based on double alpha-cut. Appl Soft Comput 98:106890

67. Wu J, Liu F, Rong Y, Liu Y, Liu C (2020) Hesitant fuzzy generalised Bonferroni mean operators based on archimedean copula for multiple-attribute decision-making. Math Probl Eng 2020:8712376. https://doi.org/10.1155/2020/8712376

68. Liu P, Xu H, Geng Y (2020) Normal wiggly hesitant fuzzy linguistic power Hamy mean aggregation operators and their application to multi-attribute decision-making. Comput Ind Eng 140:106224

69. Mahmood T, Ur Rehman U, Ali Z, Chinram R (2020) Jaccard and dice similarity measures based on novel complex dual hesitant fuzzy sets and their applications. Math Probl Eng 2020:5920432. https://doi.org/10.1155/2020/5920432

70. Wang Z, Nie H, Zhao H (2020) An extended GEDM method with heterogeneous reference points of decision makers and a new hesitant fuzzy distance formula. Comput Ind Eng 146:106533

71. Li X, Huang X (2020) A novel three-way investment decisions based on decision-theoretic rough sets with hesitant fuzzy information. Int J Fuzzy Syst 22:2708-2719

72. Karamaz F, Karaaslan F (2021) Hesitant fuzzy parameterized soft sets and their applications in decision making. J Ambient Intell Hum Comput 12:1869-1878

73. Yager RR (1988) On ordered weighted averaging aggregation operators in multicriteria decision making. IEEE Trans Syst Man Cybern 18(1):183-190

74. Dombi J (1982) A general class of fuzzy operators, the demorgan class of fuzzy operators and fuzziness measures induced by fuzzy operators. Fuzzy Sets Syst 8(2):149-163

75. Chen X, Li J, Qian L, Hu X (2016) Distance and similarity measures for intuitionistic hesitant fuzzy sets. International conference on artificial intelligence: technologies and applications (ICAITA)

76. Garg G (2018) Hesitant Pythagorean fuzzy sets and their aggregation operators in multiple attribute decision-making. Int J Uncertain Quantif 8(3):267-289

77. Yang W, Yongfeng P (2020) New q-Rung orthopair hesitant fuzzy decision making based on linear programming and TOPSIS. IEEE Access 8:221299-221311

78. Al-Husseinawi AH (2021) Hesitant T-spherical fuzzy sets and their application in decision-making. M.Sc thesis, Graduate School of Natural and Applied Sciences, Cankiri Karatekin University, Cankiri, Turkey

Publisher's Note Springer Nature remains neutral with regard to jurisdictional claims in published maps and institutional affiliations. 